Supporting Information for

\title{
Generic orbital design of higher-order topological quasicrystalline insulators with odd five-fold rotation symmetry
}

\author{
Huaqing Huang,,${ }^{* \dagger,+, \uparrow}$ Jiahao Fan, ${ }^{\dagger}$ Dexin $\mathrm{Li}^{\dagger}{ }^{\dagger}$ and Feng $\mathrm{Liu}^{*, \S}$ \\ ${ }^{\dagger}$ School of Physics, Peking University, Beijing 100871, China \\ ${ }^{\star}$ Center for High Energy Physics, Peking University, Beijing 100871, China \\ ${ }^{\mathbb{I}}$ Collaborative Innovation Center of Quantum Matter, Beijing 100871, China \\ ${ }^{\S}$ Department of Materials Science and Engineering, University of Utah, Salt Lake \\ City, Utah 84112, USA
}

E-mail: huaqing.huang@pku.edu.cn; fliu@eng.utah.edu 


\section{Contents}

I. Section S1: Numerical results of quasicrystals 3

A. Approximants of quasicrystals 3

B. Additional results of the octagonal quasicrystal $\quad 7$

C. Additional results of the pentagonal quasicrystal 8

D. Symmetry analysis of quasicrystals 9

II. Section S2: Theoretical analysis 13

A. Effective k.p model 13

B. Pseudo-time-reversal symmetry 16

C. Pseudospin Chern number for pseudo-TR-broken systems 16

D. Higher-order topology from boundary perspective $\quad 17$

E. Mirror-graded Berry phase 21

F. Calculation of the higher-order topological invariant 23

G. Discussion of fivefold quasicrystals 23

H. Robustness of topological corner states $\quad 27$

I. HOTIs induced by $p$ - $d$ band inversion in different lattices 29

III. Section S3: Higher-order topology induced by other types of band inversion 30

A. Band inversion between $d_{ \pm 1}$ and $f_{ \pm 2}$ orbitals 31

B. Band inversion between $d_{ \pm 1}$ and $d_{ \pm 2}$ orbitals 31

$\begin{array}{ll}\text { References } & 32\end{array}$ 


\section{SECTION S1: NUMERICAL RESULTS OF QUASICRYSTALS}

\section{A. Approximants of quasicrystals}

The fivefold quasicrystal lattice is constructed according to the Penrose tiling, which contains "fat" and "thin" rhombuses. Since the quasicrystal lattice possesses long-range orientational order but lacks translational symmetry, we cannot use the Bloch theorem as for the crystal calculations. However, it is still possible to generate a series of periodic lattices with a growing number of atoms that approximate the infinite quasicrystal lattice according to the quasicrystal tiling approximants [1-4].

The approximate model can be constructed in the framework of the multi-grid method, as demonstrated by de Bruijn [5]. Taking the Penrose lattice (PL) as an example, a PL tiled by two kinds of rhombuses (fat and thin) can be constructed as the dual lattices of a pentagrid. A pentagrid $\mathbf{G}$ is an ensemble of five sets of equally spaced parallel lines, and each set of parallel lines $\mathbf{G}_{j}$ is called a grid:

$\mathbf{G}=\bigcup_{j=1}^{5} \mathbf{G}_{j}, \mathbf{G}_{j}=\left\{\mathbf{x} \in R^{2} \mid \mathbf{x} \cdot \mathbf{e}_{j}=k_{j}+\gamma_{j}, k_{j} \in Z\right\}, \mathbf{e}_{j}=(\cos (j-1) \phi, \sin (j-1) \phi), \phi=2 \pi / 5$,

where $R$ and $Z$ are the sets of all real numbers and all integers, respectively. The five real grid parameters $\left\{\gamma_{j}\right\}$, satisfy the relation $\sum_{j} \gamma_{j}=0(\bmod 1)$. The five unit grid vectors $\mathbf{e}_{j}$ determine the direction and the spacing of each grid $\mathbf{G}_{j}$. The pentagrid divides the grid space into small pieces in the shape of polygons. The PL are generated by mapping these polygons into rhombus vertices in the physical space, and these two lattices are consequently dual to each other. For each piece in the grid space, let us define a set of five integer indices: $K_{j}(x)=\left\lfloor\mathbf{x} \cdot \mathbf{e}_{j}-\gamma_{j}\right\rfloor(j=1, \cdots, 5)$, where $\mathbf{x}$ is taken inside the piece and $\lfloor x\rfloor$ denotes the largest integer less than or equal to $x$. Next, assign the piece a $2 \mathrm{D}$ vector by $P(\mathbf{x})=\sum_{j=1}^{5} K_{j}(\mathbf{x}) \mathbf{t}_{j}$, then, this vector gives the coordinate of the corresponding rhombus vertex. Here, $\mathbf{t}_{j}$ are called the tiling vectors and usually chosen to be $\mathbf{t}_{j}=\mathbf{e}_{j}$.

To obtain a periodic approximant of PL, one can construct a periodic pentagrids which approximate the original one. The nonperiodicity of the PL comes from the property that any two grids in the pentagrid form a rhombus lattice, but the other three grids are incommensurate to this lattice. The specific match procedure involves (1) fixing two grids as in the original pentagrid; (2) changing the directions and the spacings of the other 
three grids so that they will be commensurate to the lattice made of the fixed two grids. As shown in Fig. S1(a), the five solid lines show the lines $\mathbf{x} \cdot \mathbf{e}_{j}=0$, and dashed lines $\mathbf{x} \cdot \mathbf{e}_{j}=1$. We fix the grids 1 and 2 , which form a rhombus lattice tiled by a unit cell OACB with the edge length $1 / \sin \phi=a$. Let $P_{j}$ and $Q_{j}$ be the intersections of the line $\mathbf{x} \cdot \mathbf{e}_{j}=1(j=3,4,5)$ with $\mathrm{OA}$ and $\mathrm{OB}$, respectively. In the case of the original pentagrid, $O P_{3}=O P_{4}=O Q_{4}=O Q_{5}=\tau a, O P_{5}=O Q_{3}=a$. Here, the irrational golden ratio $\tau$ is the reason that the grids $j=3,4,5$ are incommensurate with respect to the rhombus lattice made of grids 1 and 2. Therefore a periodic approximation of the pentagrid can be obtained by substituting rational numbers for the $\tau$ 's, and this approximation is explicitly expressed by the grid vectors,

$$
\tilde{\mathbf{e}}_{1}=\mathbf{e}_{1}, \tilde{\mathbf{e}}_{2}=\mathbf{e}_{2}, \tilde{\mathbf{e}}_{3}=-\mathbf{e}_{1}+\frac{1}{r_{3}} \mathbf{e}_{2}, \tilde{\mathbf{e}}_{4}=-\frac{1}{s_{4}} \mathbf{e}_{1}-\frac{1}{r_{4}} \mathbf{e}_{2}, \tilde{\mathbf{e}}_{5}=\frac{1}{s_{5}} \mathbf{e}_{1}-\mathbf{e}_{2},
$$

where the $r$ 's and s's are rational approximants of $\tau$ in the coefficients of OP's and OQ's, respectively. The grid spacing for $j=3,4,5$ is no longer 1 after the approximation. Now having the periodic pentagrids, one can generate periodic approximations of the PL by following the mapping procedure explained above. The periodic approximations of the lattice are determined by the approximants of $\tau$. The optimal approximants are the ratios of successive Fibonacci numbers $\tau \tau_{n}=F_{n+1} / F_{n}$. Here, $\left\{F_{n}\right\}$ is defined by the recursion relation $F_{n+1}=F_{n}+F_{n-1}$ with the initial values $F_{1}=1, F_{2}=2$. As $n$ increases, the approximant approaches the golden ratio rapidly after initial oscillation [see Fig. S1(b)]. Thus $\tau$ can be systematically approximated by the sequence $\left\{\tau_{n}\right\}=\{2,3 / 2,5 / 3,8 / 5,13 / 8,21 / 13, \cdots\}$. Then one can generate a sequence of the periodic approximations by substituting $\tau_{n}$ for all r's and s's above. This choice provides periodic lattices with unit cells in the shape of a thick Penrose rhombus, as shown in Fig. S1(c) and S2(d). The periodic approximate models have the following properties: (1) the periodic boundary condition can be imposed on the unit cell in both directions; (2) there are no extra modulations for the pentagrid.

Similarly, for the Ammann-Beenker tiling respecting perfect eight-fold rotational symmetry [6-9], one can construct the approximate model using the multi-grid method. However, for the ease of numerical calculation, we adopt an iterative subdivision algorithm. The Ammann-Beenker tiling consists of two primitive tiles: a rhombus with angles $\pi / 4$ and $3 \pi / 4$, and the square. An iterative subdivision algorithm can be used to construct large patches of this quasicrystal, as shown in Fig. S2. The construction procedure contains three 


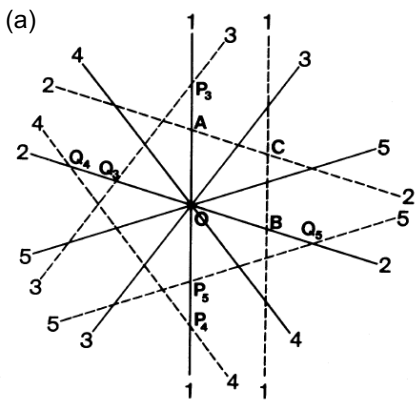

(b)

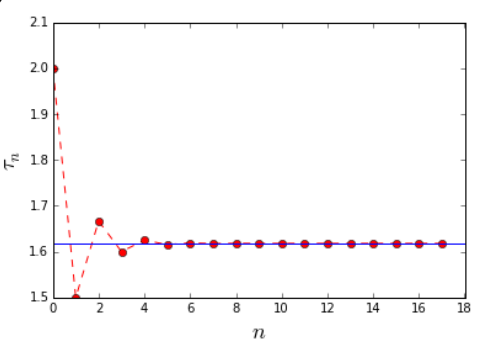

(c)

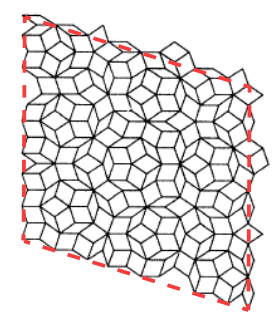

(d)

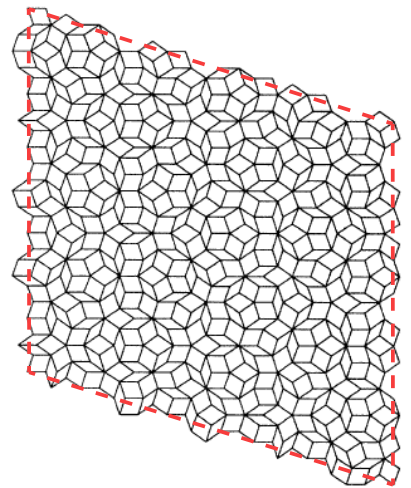

Figure S1: Quasicrystal approximants of the Penrose lattice. (a) Central part of the pentagrid when all $\gamma_{j}=0$. (b) The golden ratio $\tau$ is approximated by the ratio of successive Fibonacci numbers $\tau_{n}=F_{n+1} / F_{n}$. Unit cells of the periodic Penrose lattices: (c) $\tau_{n}=5 / 3, N=199 ;$ (d) $\tau_{n}=8 / 5, N=521$.

steps: the initial step of the algorithm consists of converting a square shape into two decorated triangles, as shown in Fig. S2(a). Afterwards, one iteratively applies the subdivision rules shown in Fig. S2(a) for $N$ times, each time obtaining a quasicrystal patch with a larger number of sites. Finally, at the end, one converts all pairs of decorated triangles back into squares, by applying the equivalence of Fig. S2(a). This procedure guarantees that an initially eight-fold symmetric tiling will retain the eight-fold symmetry after any number of iterations. Examples of tilings obtained after $N=1$ and $N=2$ applications of the subdivision process to the initial square and octagonal tilings are shown in Fig. S2(b) and Fig. S2(c), respectively. Apparently, the obtained square patches can be regarded as the unit cell of periodic approximants, which approach the exact quasicrystal limit with an increasing number of subdivision procedures.

Figure S3 shows a series of periodic approximants with growing unit cells. These approximants can approach the infinite Ammann-Beenker-type quasicrystal gradually. Figure S4 
(a)

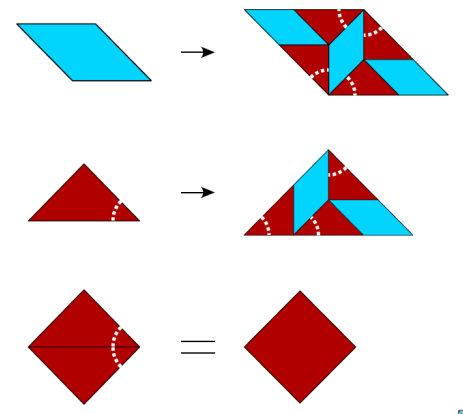

(b)
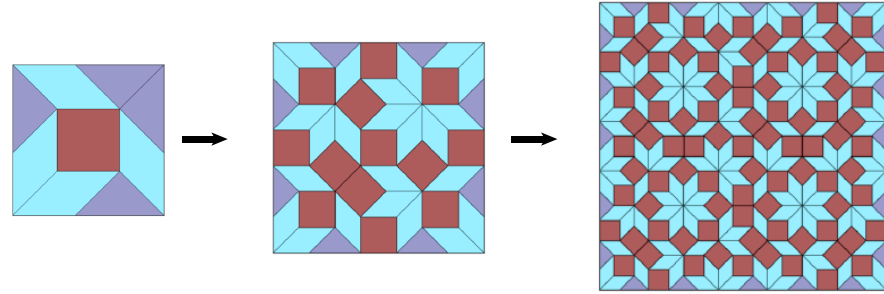

(c)

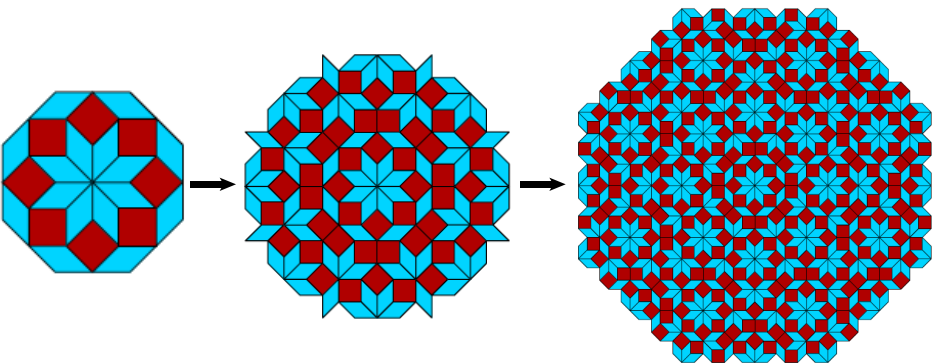

Figure S2: Obtaining the Ammann-Beenker tiling through subdivision. (a) Elementary step of the subdivision of the rhombus and the (decorated) triangle and the Equivalence between two triangles and a square tile, which is applied in the beginning and the end of the subdivision process. (b) Example of the subdivision process applying to the Initial (b) square and (c) octagonal tilings, respectively.

shows the band structures of several approximant phases of the Ammann-Beenker-type quasicrystal. The double band inversion between conduction and valence bands, which are dominated by $\left(p_{x}, p_{y}\right)$ and $\left(d_{x^{2}-y^{2}}, d_{x y}\right)$ orbitals respectively, occurs around the central $\Gamma$ point of the Brillouin zone (BZ). With the increasing approximant size, the corresponding BZ shrinks, and the calculated band structures attain gradually denser energy levels. However, the essential feature of the $p$ - $d$ band inversion is still visible from the orbital contribution of states around the energy gap. 
(a)
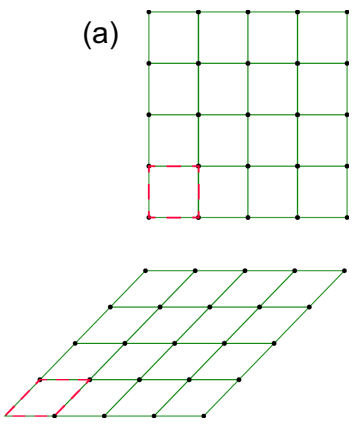

(e)

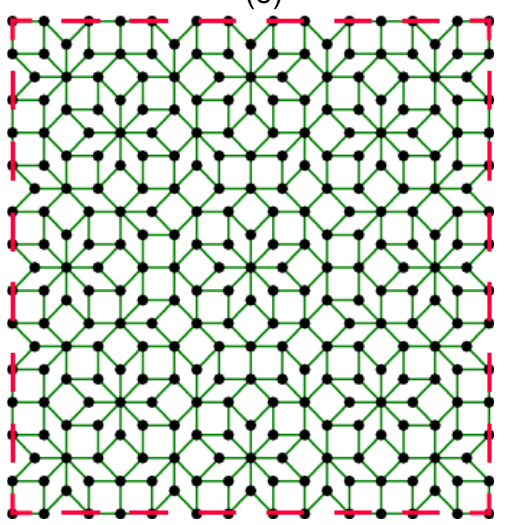

(b)

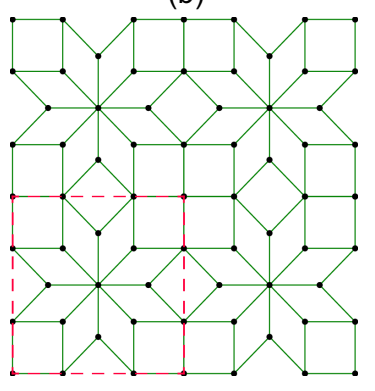

(c)

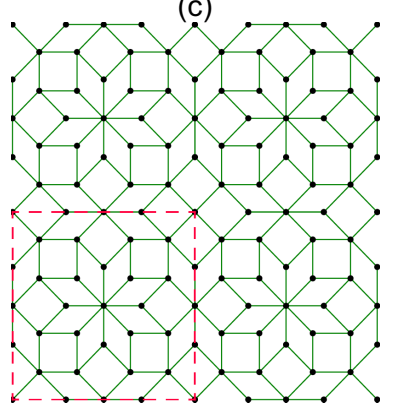

(d)

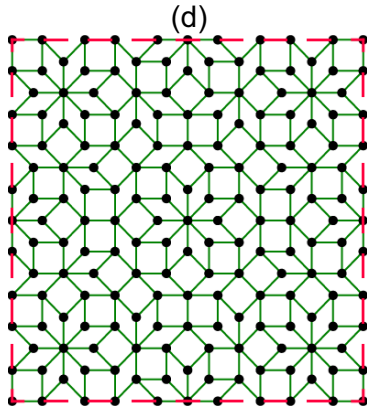

(g) (f)

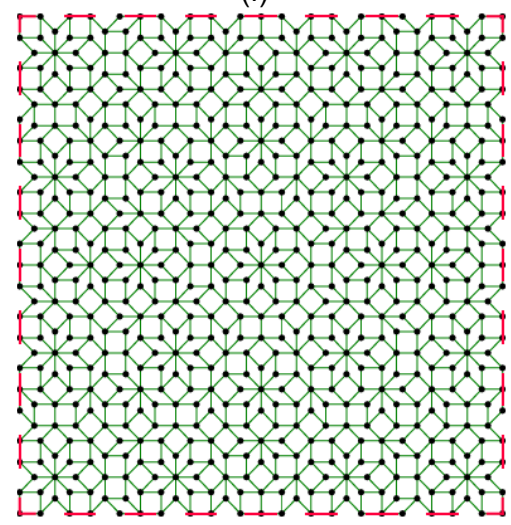

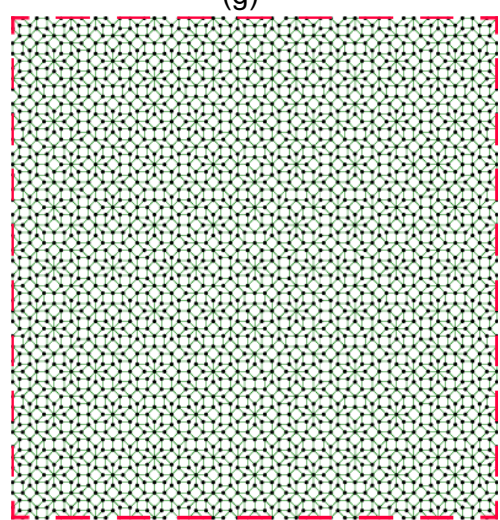

Figure S3: Quasicrystal approximants of the Ammann-Beenker-type quasicrystal. The red dashedline boxes indicate the unit cell for these approximants.

\section{B. Additional results of the octagonal quasicrystal}

Figure S5 shows the energy spectrum of the octagonal sample of the eightfold quasicrystal. There are eight corner states around the Fermi level (marked in red). The spatial charge distribution of all eight states in the bottom panel of Fig. S5 clearly shows that they are well localized at corners. It is noted that there exists slight energy splitting of these corner states. Due to the finite size of the sample, there are weak couplings between adjacent corner states. This leads to the energy splitting and the recombination of corner states. These corner-state results are also reproducible from a simple tight-binding (TB) Hamiltonian, as shown in Fig. S6. Within this model, the energy levels of eight coupled corner states are composed of a minimum, a maximum, and three pairs of double degenerates levels in between. And the resulting corner states from this model can be considered as linear combinations of eight isolated corner states. Both the energy levels and corner states obtained from the TB model are consistent with our results in Fig. S5. As the Fermi level is determined by the half-filling of the whole energy spectrum, we find that five of the eight corner states are occupied. If 

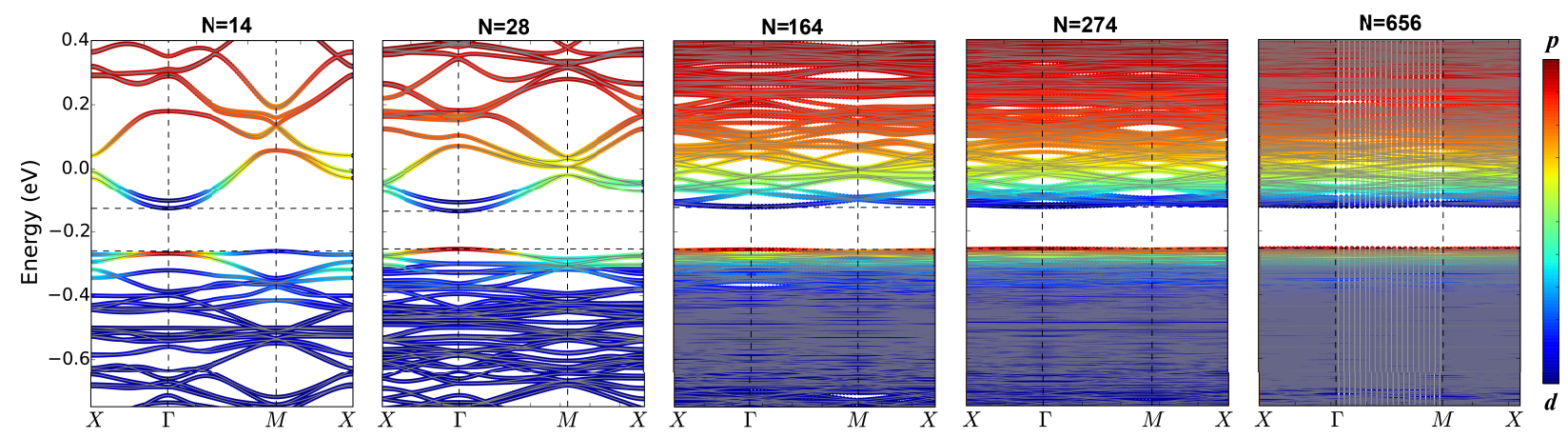

Figure S4: Band structures of quasicrystal approximants. Orbital-resolved band structures of several periodic approximants with increasing lattice size, as shown in Fig. S3(b)-(f). A $p$ - $d$ band inversion between conduction and valence bands (dominated by $\left(p_{x}, p_{y}\right)$ and $\left(d_{x^{2}-y^{2}}, d_{x y}\right)$ respectively) occurs around $\Gamma$.

electron filling is fine tuned to occupy all corner modes, $3 / 8$ electron is localized at each corner, leading to a fractionalized charge distribution.

\section{Additional results of the pentagonal quasicrystal}

Figure S7 shows the energy spectrum of the pentagonal sample of the Penrose-type fivefold quasicrystal. There are five corner states near the Fermi level. The spatial distribution of all corner states are presented in bottom panels of Fig. S7. Due to the finite-size effect, these corner states interact and hybridize with each other. Such interactions also lead to slight splitting between the degenerate corner states. A simplified TB model is adopted to explain the recombination of corner states, as shown in Fig. S8. Within this model, corner states have tiny couplings $(t)$ between them. The resulting corner states from this simplified model are linear combinations of five isolated corner states. This is consistent with the direct calculations of the Penrose-type quasicrystal (see Fig. S7). As the energy levels of corner states split into two double degenerate pairs and a single maximum, a fractional charge of $2 / 5$ or $3 / 5$ electron per corner can be realized by fine tuning the electron occupation of these corner modes. 


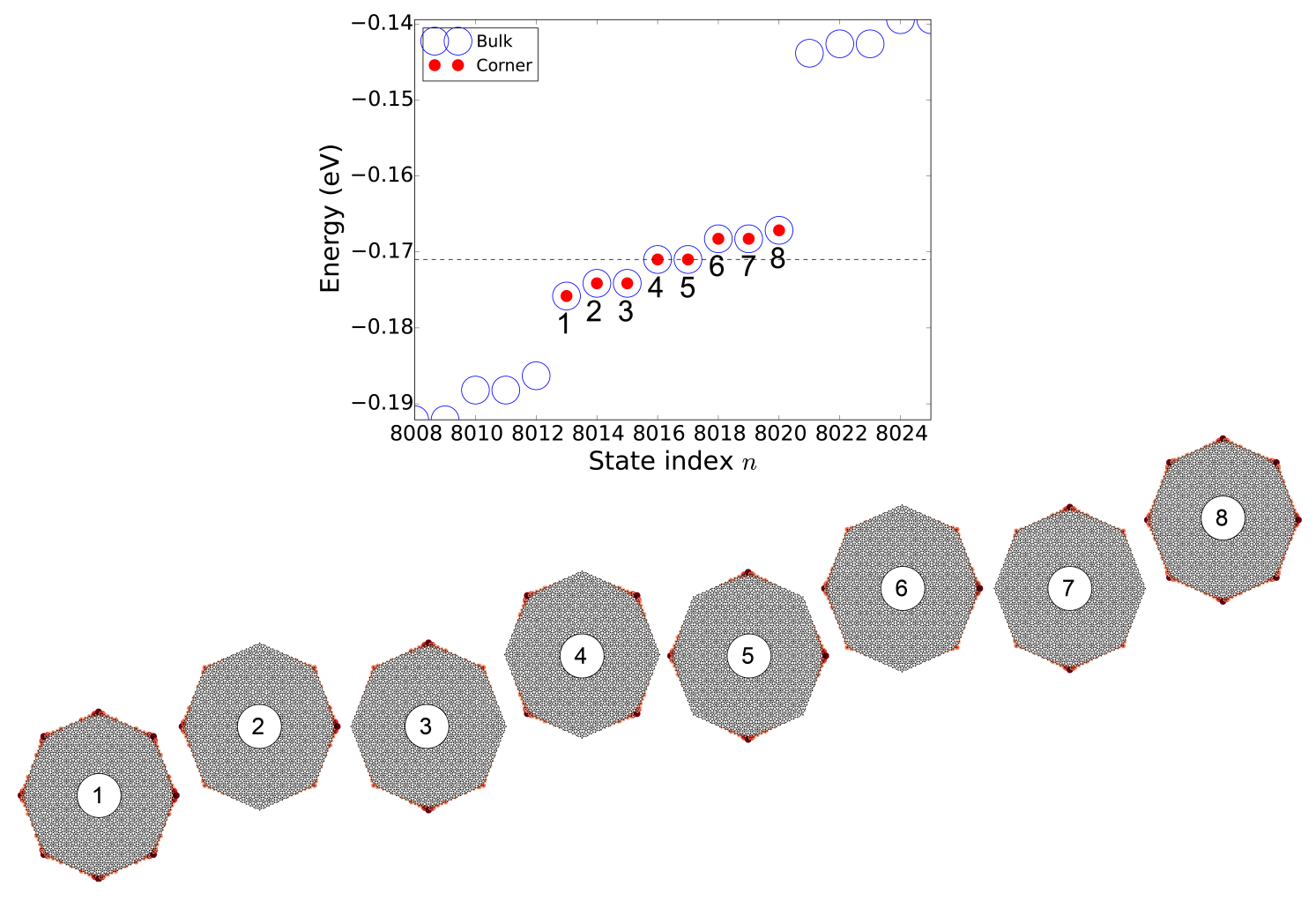

Figure S5: Eight corner states in a symmetric octagonal sample of the eightfold Ammann-Beenkertype quasicrystal. The parameters used here are $\epsilon_{p}=0.5, \epsilon_{d}=-0.5, V_{p p \sigma}=-0.392, V_{p p \pi}=$ $-0.392, V_{d d \sigma}=0.098, V_{d d \pi}=0.235, V_{d d \delta}=0.333, V_{p d \sigma}=0.216, V_{p d \pi}=0.216 \mathrm{eV}$.

TABLE S1: Character table for the $C_{5 v}$ point group

\begin{tabular}{c|cccc|c}
\hline \hline$C_{5 v}$ & $E$ & $2 C_{5}$ & $2 C_{5}^{2}$ & $5 \sigma_{v}$ & bases \\
\hline$A_{1}$ & 1 & 1 & 1 & 1 & $p_{0} ; d_{0}$ \\
$A_{2}$ & 1 & 1 & 1 & -1 & $h_{5}-h_{-5}$ \\
$E_{1}$ & 2 & $2 \cos (2 \pi / 5)$ & $2 \cos (4 \pi / 5)$ & 0 & $p_{ \pm 1} ; d_{ \pm 1}$ \\
$E_{2}$ & 2 & $2 \cos (4 \pi / 5)$ & $2 \cos (2 \pi / 5)$ & 0 & $d_{ \pm 2} ; f_{ \pm 2} ; f_{ \pm 3}$ \\
\hline \hline
\end{tabular}

\section{Symmetry analysis of quasicrystals}

The character tables of $C_{5 v}$ and $C_{8 v}$ point groups are listed in Table S1 and S2, respectively. The doubly degenerate atomic orbitals $p_{ \pm 1}=\frac{1}{\sqrt{2}}\left(p_{x} \pm i p_{y}\right)$ with $l=1$ and $d_{ \pm 2}=\frac{1}{\sqrt{2}}\left(d_{x^{2}-y^{2}} \pm i d_{x y}\right)$ with $l=2$ belong to the $2 \mathrm{D}$ irreducible representation $E_{1}$ and $E_{2}$ respectively, and therefore, are chosen as bases functions for the realization of the double 


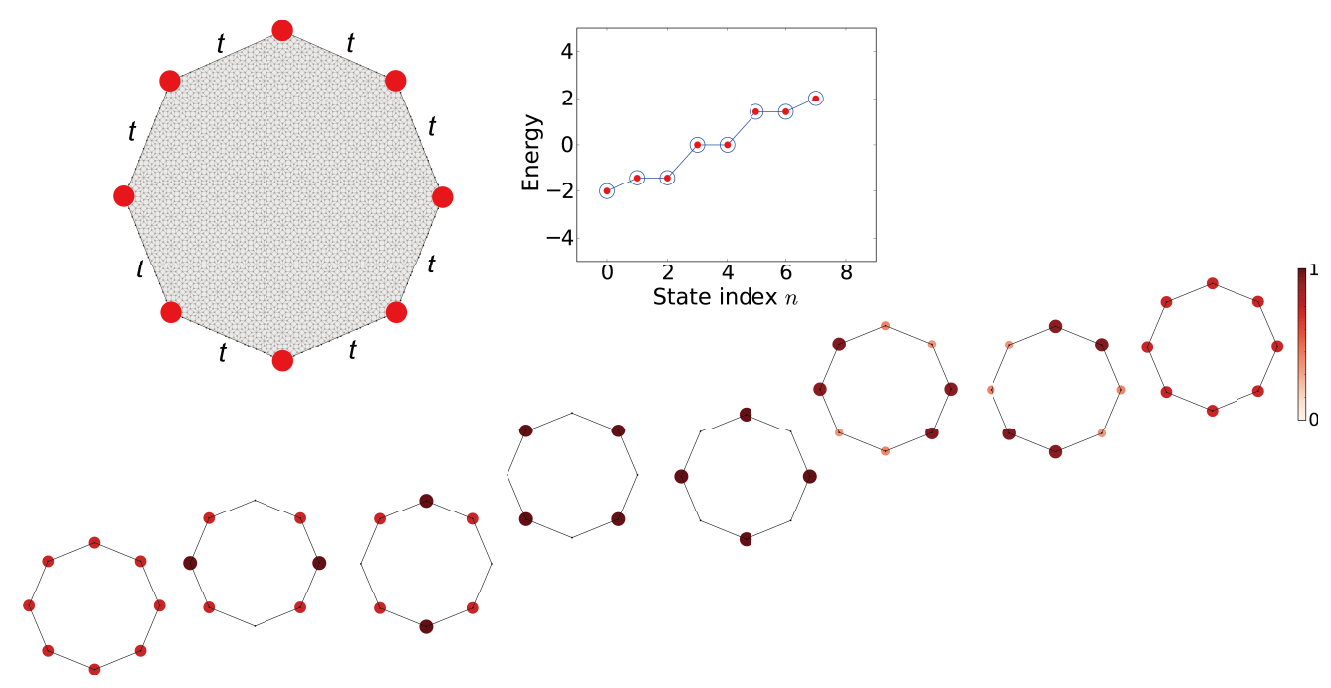

Figure S6: Schematic illustration of the hybridization of corner states in a finite octagonal sample of eightfold quasicrystals. Due to the finite-size-effect-induced weak coupling $t$ between adjacent corners, new corner states which can be considered as linear combinations of eight isolated corner states are formed. There exists slight energy splitting for these new corner states, which is exponentially suppressed with the increasing sample size. The spatial distributions of the corner states are consistent with those presented in Fig. S5.
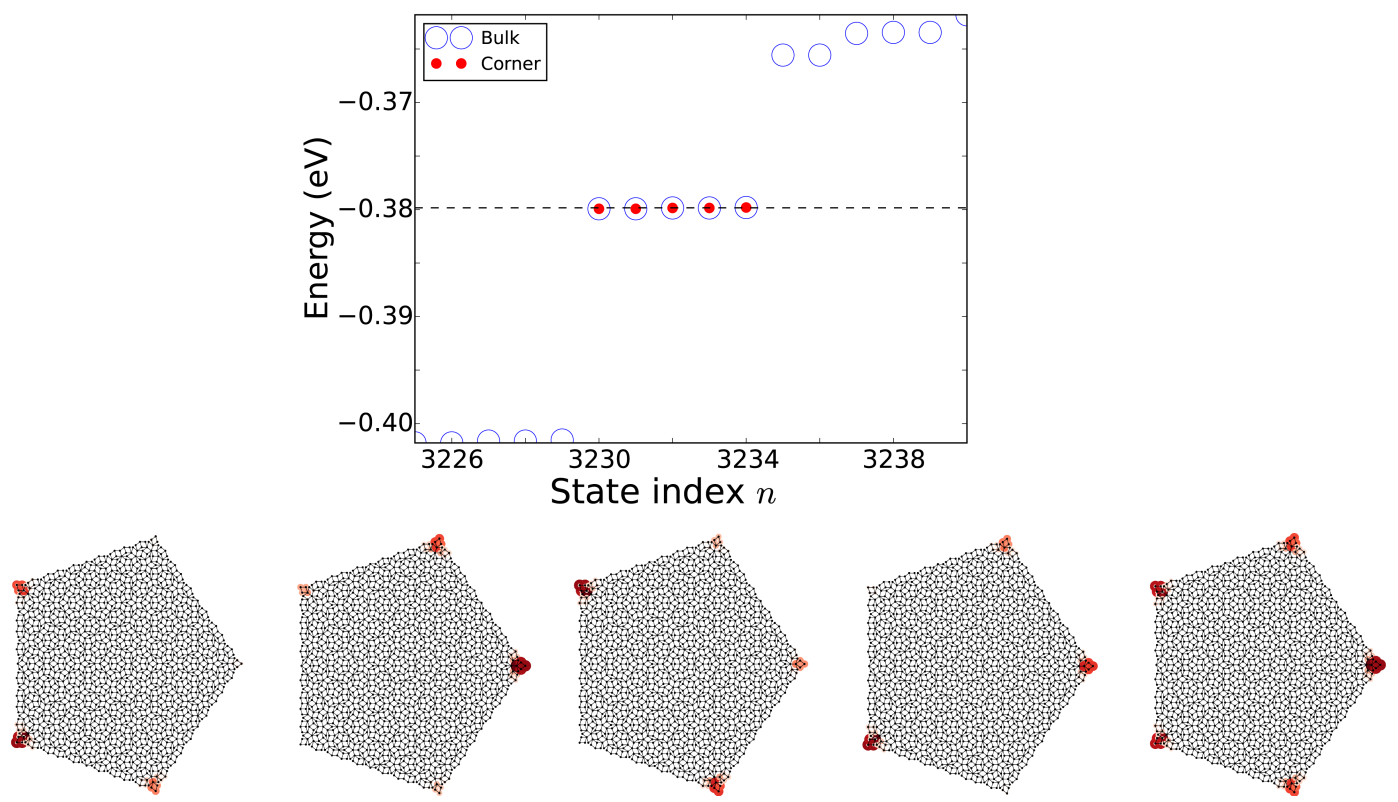

Figure S7: Five corner states (red dots) in a symmetric pentagonal sample of the Penrose-type fivefold quasicrystal. The parameters used here are $\epsilon_{p}=0.5, \epsilon_{d}=-0.5, V_{p p \sigma}=-0.2, V_{p p \pi}=$ $-0.1, V_{d d \sigma}=0.4, V_{d d \pi}=0.02, V_{d d \delta}=0.15, V_{p d \sigma}=0.1, V_{p d \pi}=0.1 \mathrm{eV}$. 


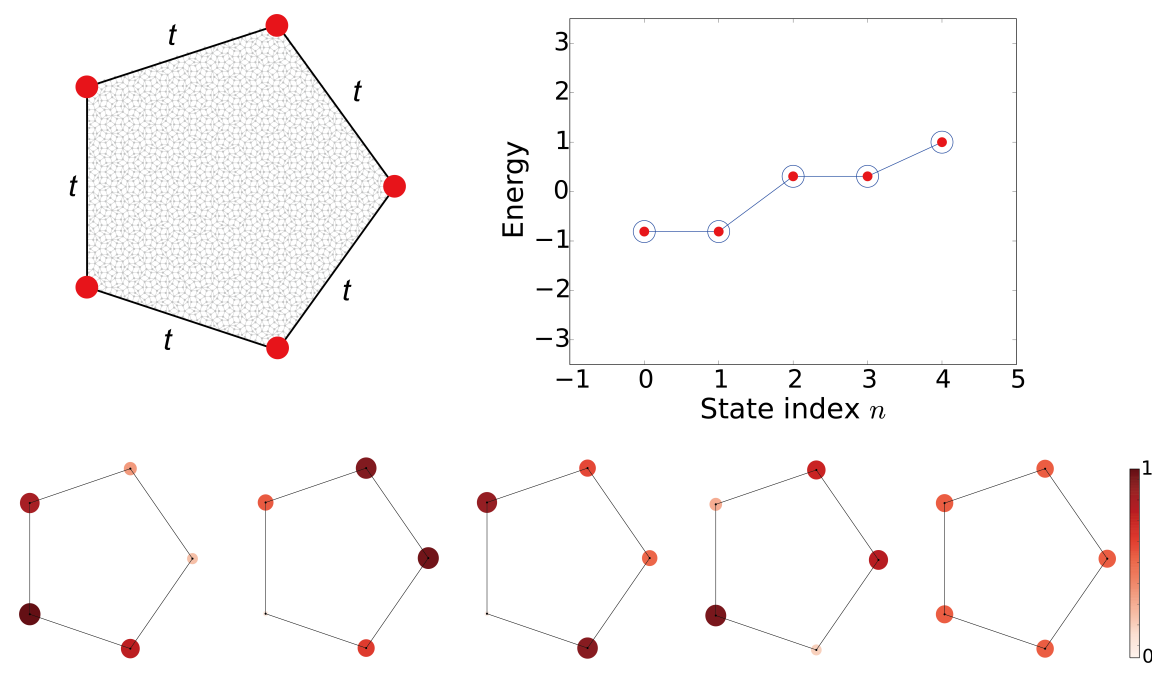

Figure S8: Schematic illustration of the hybridization of corner states in a finite pentagonal sample of fivefold quasicrystals. Due to the finite-size-effect-induced weak coupling $t$ between adjacent corner states, new corner states which can be considered as linear combinations of eight isolated corner states are formed. The spatial distributions of the corner states are consistent with those presented in Fig. S7.

TABLE S2: Character table for the $C_{8 v}$ point group

\begin{tabular}{c|ccccccc|c}
\hline \hline$C_{8 v}$ & $E$ & $2 C_{8}$ & $2 C_{4}$ & $2 C_{8}^{3}$ & $C_{2}$ & $4 \sigma_{v}$ & $4 \sigma_{d}$ & bases \\
\hline$A_{1}$ & 1 & 1 & 1 & 1 & 1 & 1 & 1 & $p_{0} ; d_{0}$ \\
$A_{2}$ & 1 & 1 & 1 & 1 & 1 & -1 & -1 & - \\
$B_{1}$ & 1 & -1 & 1 & -1 & 1 & 1 & -1 & $g_{4}+g_{-4}$ \\
$B_{2}$ & 1 & -1 & 1 & -1 & 1 & -1 & 1 & $g_{4}-g_{-4}$ \\
$E_{1}$ & 2 & $2 \cos (\pi / 4)$ & 0 & $-2 \cos (\pi / 4)$ & -2 & 0 & 0 & $p_{ \pm 1} ; d_{ \pm 1}$ \\
$E_{2}$ & 2 & 0 & -2 & 0 & 2 & 0 & 0 & $d_{ \pm 2} ; f_{ \pm 2}$ \\
$E_{3}$ & 2 & $-2 \cos (\pi / 4)$ & 0 & $2 \cos (\pi / 4)$ & -2 & 0 & 0 & $f_{ \pm 3} ; g_{ \pm 3}$ \\
\hline \hline
\end{tabular}

band inversion in pentagonal and octagonal quasicrystals. Other orbitals can also be used to realize double band inversion, which will be discussed in the last section.

The fractional quantization of corner charge arises form a filling anomaly [10-12] when corners are induced in $C_{n}$-symmetric systems. That is, the topological corner charge is the consequence of charge redistribution due to the mismatch between the positions of ionic charge (atomic positions) and the "Wannier centers" of occupied states. For trivial insula- 
tors without double band inversion, the occupied bands can be adiabatically connected to the atomic limit without energy gap closure or $C_{n}$-symmetry breaking (e.g., by gradually increasing the interatomic distance). Consequently, the Wannier centers are always consistent with atomic positions, and there is no topological corner charge. In contrast, for nontrivial HOTI systems, due to the double band inversion at the center of the Brillouin zone, occupied states cannot smoothly connect to the atomic limit while maintaining $C_{n}$ symmetry, i.e., a mismatch arises between atomic positions and Wannier centers. In general, the topological corner charge depends on the location of the "Wannier center" but not on the detailed shape of Wannier functions as long as the rotation symmetry is properly implemented [11].

Specifically, for crystals with $C_{n}$ symmetry, the charges are equal among the corners, given by[12]:

$$
Q_{\text {corner }}=Q_{c_{-} 1 a}^{(n)}=\frac{\left(n_{1 a}^{(\text {ion })}-n_{1 a}^{(e)}\right)}{n}|e| \quad(\bmod |e|),
$$

where $1 a$ is the Wyckoff position with $C_{n}$ symmetry located at the center of the crystal. $n_{1 a}^{(\text {ion })}|e|$ is the ionic charge at $1 a$, and $n_{1 a}^{(e)}$ is the number of electronic Wannier functions at $1 a$. For trivial insulators, Wannier centers are located at the center of the unit cell, coinciding with the position of the ionic centers; while for nontrivial insulators with double band inversion, Wannier centers pinned, by symmetry, to Wyckoff position other than the center of the unit cell. For systems with $C_{3}, C_{4}$ and $C_{6}$, the above formula can be further simplified based on the rotation eigenvalues of the occupied bands, as shown in Refs. [10-12]. Importantly, the general idea of filling anomaly is still valid for $C_{n}$-symmetric systems without translational symmetry, albeit with different distribution of topological corner charges. Namely, when the double band inversion occurs in $C_{n}$-symmetric systems, the Wannier centers of occupied states are no longer consistent with atomic positions, giving rise to the topological corner charges.

Taking the octagonal quasicrystal as an example, the rotation operator is

$$
\hat{C}_{8}=\exp \left(-i \frac{\pi}{4} \sigma\right) \mathcal{R}
$$

where $\sigma$ is the rotation matrix in the orbital space and $\mathcal{R}$ is an orthogonal matrix permuting the sites of the quasicrystal to rotate the whole system by an angle of $\pi / 4$. The eigenvalue of $\hat{C}_{8}$ is $\omega_{p}=e^{i \pi p / 4}, \quad(p=0,1,2, \cdots, 7)$. As shown in Table $\mathrm{S} 2, p_{ \pm 1}=\frac{1}{\sqrt{2}}\left(p_{x} \pm i p_{y}\right)$ and $d_{ \pm 2}=\frac{1}{\sqrt{2}}\left(d_{x^{2}-y^{2}} \pm i d_{x y}\right)$ orbitals belong to different $2 \mathrm{D}$ irreducible representations of the $C_{8 v}$ group. For our proposed model, one can calculate the indices $\chi^{(8)}=n_{ \pm 1}, n_{ \pm 2}$ ) for 
occupied states to determine its higher-order topology, where $n_{ \pm p}$ is the number of states with the eigenvalue $\omega_{p}$ or $\omega_{8-p}$. As a reference, we fist calculate $\chi_{0}^{(8)}$ for the trivial atomic limit without band inversion. For any $C_{8 v}$-symmetric quasicrystals, a $\chi^{(8)}$, different from $\chi_{0}^{(8)}$, emerges to signal an obstructed atomic limit, implying the existence of topological corner charges. Same analyses are also applicable to pentagonal quasicrystals according to the character table of $C_{5 v}$ (see Table S1).

\section{SECTION S2: THEORETICAL ANALYSIS}

\section{A. Effective k.p model}

As an illustrative example, we consider the simplest periodic approximant - a square lattice. The generic atomic basis TB model with four orbitals $\left(p_{x}, p_{y}, d_{x y}, d_{x^{2}-y^{2}}\right)$ per site on a square lattice is given by

$$
\begin{aligned}
& H=\left(\begin{array}{cc}
H_{p} & H_{p d} \\
H_{p d}^{\dagger} & H_{d}
\end{array}\right) \\
& H_{p}=\left(\begin{array}{cc}
\epsilon_{p}+2 V_{p p \sigma} \cos k_{x}+2 V_{p p \pi} \cos k_{y} & 0 \\
0 & \epsilon_{p}+2 V_{p p \sigma} \cos k_{y}+2 V_{p p \pi} \cos k_{x}
\end{array}\right) \text {, } \\
& H_{d}=\left(\begin{array}{cc}
\epsilon_{d}+\left(\frac{3}{2} V_{d d \sigma}+\frac{1}{2} V_{d d \delta}\right)\left(\cos k_{x}+\cos k_{y}\right) & 0 \\
0 & \epsilon_{d}+2 V_{d d \pi}\left(\cos k_{x}+\cos k_{y}\right)
\end{array}\right), \\
& H_{p d}=\left(\begin{array}{cc}
\sqrt{3} i V_{p d \sigma} \sin k_{x} & 2 i V_{p d \pi} \sin k_{y} \\
-\sqrt{3} i V_{p d \sigma} \sin k_{y} & 2 i V_{p d \pi} \sin k_{x}
\end{array}\right) \text {. }
\end{aligned}
$$

For this spinless model, we can take the linear combination of orbitals with positive and negative orbital angular momentum (magnetic quantum number) as the up and down pseudospin states, i.e.,

$$
p_{ \pm}=\frac{1}{\sqrt{2}}\left(p_{x} \pm i p_{y}\right), \quad d_{ \pm}=\frac{1}{\sqrt{2}}\left(d_{x^{2}-y^{2}} \pm i d_{x y}\right) .
$$


Consequently, by performing a basis transformation from $\left(p_{x}, p_{y}, d_{x^{2}-y^{2}}, d_{x y}\right) \rightarrow$ $\left(p_{+}, d_{+}, p_{-}, d_{-}\right)$via the transformation matrix

$$
U=\frac{1}{\sqrt{2}}\left(\begin{array}{cccc}
1 & i & 0 & 0 \\
0 & 0 & 1 & i \\
1 & -i & 0 & 0 \\
0 & 0 & 1 & -i
\end{array}\right)
$$

the Hamiltonian Eq. (5) transfers to

$$
H^{\prime}=U H U^{\dagger}=\left(\begin{array}{cc}
H_{+} & \Delta \\
\Delta^{\dagger} & H_{-}
\end{array}\right)
$$

where

$$
\begin{aligned}
H_{ \pm} & =\left(\begin{array}{cc}
\epsilon_{p}+\left(V_{p p \pi}+V_{p p \sigma}\right)\left(\cos k_{x}+\cos k_{y}\right) & \left(V_{p d \pi}+\frac{\sqrt{3}}{2} V_{p d \sigma}\right)\left(i \sin k_{x} \pm \sin k_{y}\right) \\
\left(V_{p d \pi}+\frac{\sqrt{3}}{2} V_{p d \sigma}\right)\left(-i \sin k_{x} \pm \sin k_{y}\right) & \epsilon_{d}+\left(\frac{1}{4} V_{d d \delta}+V_{d d \pi}+\frac{3}{4} V_{d d \sigma}\right)\left(\cos k_{x}+\cos k_{y}\right)
\end{array}\right) \\
\Delta= & \left(\begin{array}{cc}
-\left(V_{p p \pi}-V_{p p \sigma}\right)\left(\cos k_{x}-\cos k_{y}\right) & \left(V_{p d \pi}-\frac{\sqrt{3}}{2} V_{p d \sigma}\right)\left(-i \sin k_{x}-\sin k_{y}\right) \\
\left(V_{p d \pi}-\frac{\sqrt{3}}{2} V_{p d \sigma}\right)\left(i \sin k_{x}+\sin k_{y}\right) & \left(\frac{1}{4} V_{d d \delta}-V_{d d \pi}+\frac{3}{4} V_{d d \sigma}\right)\left(\cos k_{x}+\cos k_{y}\right)
\end{array}\right)
\end{aligned}
$$

To derive the effective k.p Hamiltonian, we expand the Eq. (11) to the second order in $\mathbf{k}$ and neglect the off-diagonal coupling $\Delta$ (i.e., assuming that $d_{ \pm}$orbitals are degenerate at $\Gamma$ : $V_{d d \pi}=\frac{3}{4} V_{d d \sigma}+\frac{1}{4} V_{d d \delta}$ and $\left.V_{p p \pi}=V_{p p \sigma}\right)$,

$$
H_{k . p}(k)=H_{0}(k)+\left(\begin{array}{cccc}
M-B k^{2} & A\left(i k_{x}+k_{y}\right) & 0 & 0 \\
A\left(-i k_{x}+k_{y}\right) & -M+B k^{2} & 0 & 0 \\
0 & 0 & M-B k^{2} & A\left(i k_{x}-k_{y}\right) \\
0 & 0 & A\left(-i k_{x}-k_{y}\right) & -M+B k^{2}
\end{array}\right) \text {, }
$$

where $H_{0}$ represents an overall energy shift, $M, B, A$ are parameters, $k^{2}=k_{x}^{2}+k_{y}^{2}, k_{ \pm}=$ $k_{x} \pm i k_{y}$. As the effective k.p model captures the essential band-inversion physics around the center of the BZ, one expects that it is capable of capturing the essential features of the $p$ - $d$ band inversion in other lattices, regardless of the lattice symmetry and details of band dispersion away from $\Gamma$. As we will show later, similar higher-order topology indeed exists on other periodic lattices with different symmetries (such as rhombic, trigonal, and honeycomb lattices shown in Figs. S17-S19) as well as quasicrystals. 

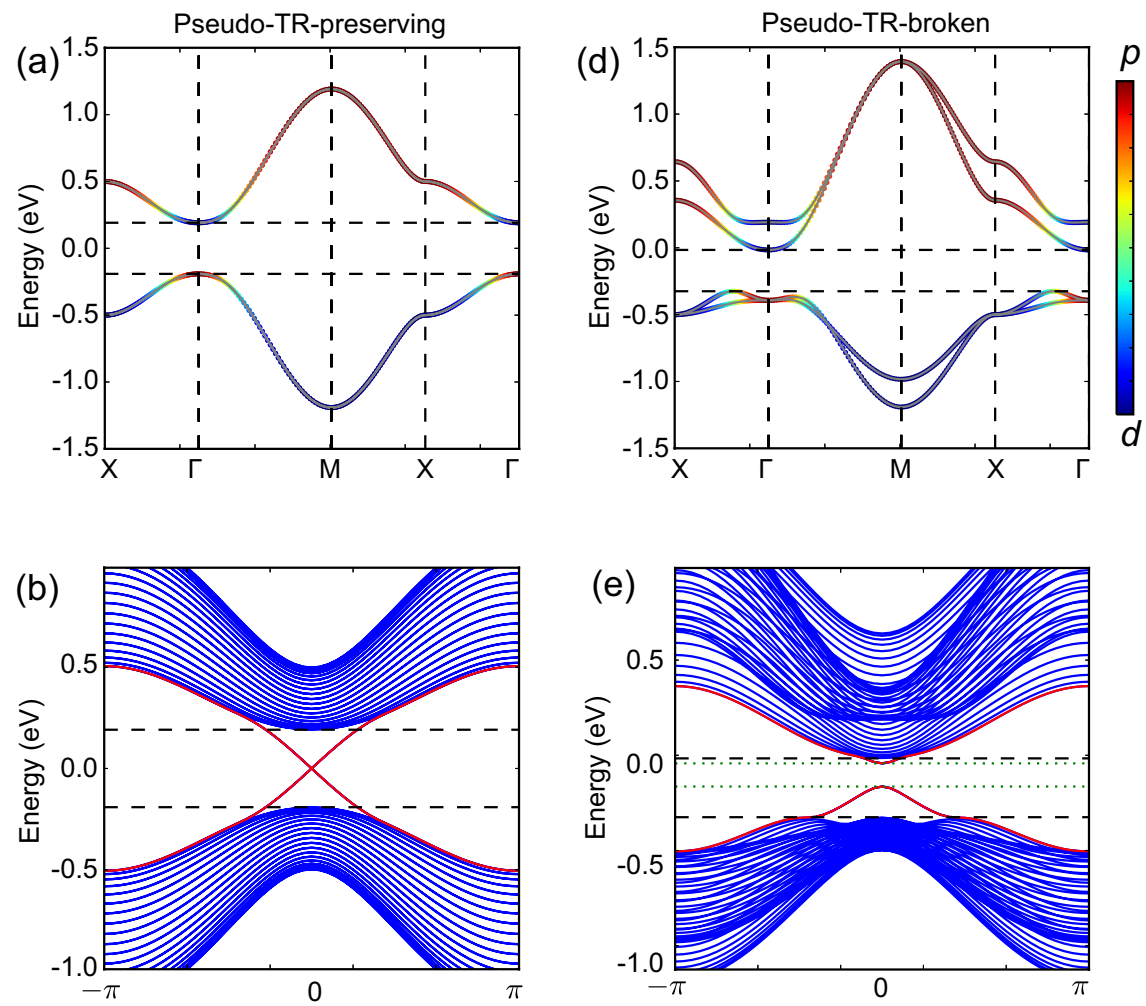

(c)

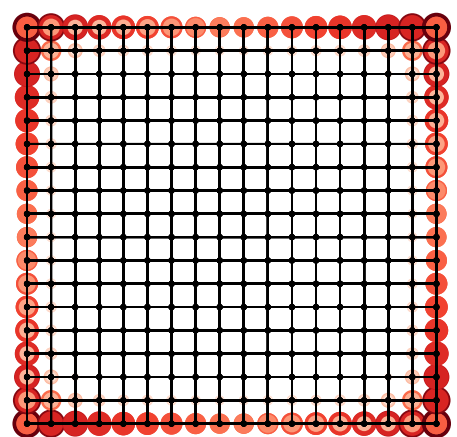

(f)

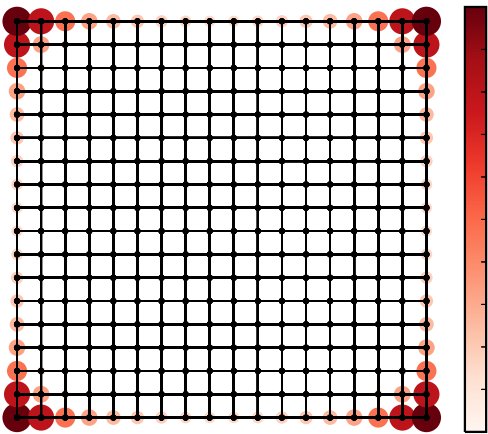

Figure S9: Electronic structures of the effective BHZ-like model with $(a, b, c)$ and without $(\mathrm{d}, \mathrm{e}, \mathrm{f})$ pseudo-TR symmetry. The parameters for the model without (with) pseudo-TR-broken term are $\epsilon_{p}=0.5, \epsilon_{d}=-0.5, V_{p p \sigma}=-0.173, V_{p p \pi}=-0.173(-0.144), V_{d d \sigma}=0.144, V_{d d \pi}=$ $0.173(0.124), V_{d d \delta}=0.259, V_{p d \sigma}=0.173, V_{p d \pi}=0.150(0.135) \mathrm{eV}$. (a,d) Bulk band structure where a double band inversion occurs around $\Gamma$. The degenerate conduction-band states at $\Gamma$ split after the pseudo-TR symmetry is broken. (b,e) Energy spectrum of nanoribbon structures. The gapless edge states in the pseudo-TR-preserving system are gapped when the pseudo-TR-breaking term is considered. (c,f) The spatial distribution of boundary states of finite square nanodisks. The edge states extend along the perimeter in the pseudo-TR-preserving system. The states appearing in the edge gap are localized at corners in the presence of the pseudo-TR-breaking term. 


\section{B. Pseudo-time-reversal symmetry}

Since the effective k.p Hamiltonian $H_{k . p}$ in Eq. (14) is in a similar form as the BernegivHughes-Zhang model for the quantum spin Hall (QSH) effect in CdTe/HgTe/CdTe quantum wells [13], the system, in principle, can host a QSH-like effect accompanied by gapless edge states due to the double band inversion mechanism. Like the QSH states that are protected by time-reversal (TR) symmetry, the QSH-like state described by the above k.p model is protected by a pseudo-TR symmetry $\mathcal{T}=i \sigma_{z} \otimes \tau_{0} \mathcal{K}$ on the basis $\left(p_{+}, d_{+}, p_{-}, d_{-}\right)$, where $\mathcal{K}$ is the complex conjugation operator and $\sigma_{z}$ is the third Pauli matrix for pseudospin. The unitary part of this pseudo-TR operator is usually represented by crystalline rotational symmetry operators as the pseudospins are emulated by orbital angular momenta with positive or negative magnetic quantum numbers.

However, we need to emphasize that the off-diagonal coupling $\Delta$ between two pseudospin submatrix is, in general, not zero, which leads to the breaking of the pseudo-TR symmetry and mixing between two pseudospin channels. This is also seen from the fact that only $p$-derived states are degenerate at $\Gamma$, while $d$-like states which belong to different irreducible representations of the space group, are not degenerate (i.e., a pseudo-Kramers degeneracy is not guaranteed), as shown in Fig. S9 and Fig. 2(a) in the main text. On the other hand, even though $\Delta$ is negligible in some situations (e.g. in trigonal lattices [14]), the pseudo-TR symmetry is broken at the edge due to the reduction of crystalline rotation symmetry at the boundary. As a consequence, the topological edge states are generally gapped (see Fig. S9).

\section{Pseudospin Chern number for pseudo-TR-broken systems}

Similar to the TR-broken QSH effect, we can calculate the pseudospin Chern number for the pseudo-TR-broken system. As the pseudospin operator is

$$
\mathcal{O}_{z}=\sigma_{z} \otimes \tau_{0}
$$

in the basis $\left(p_{+}, d_{+}, p_{-}, d_{-}\right)$with $\sigma_{x, y, z}$ and $\tau_{0}$ being Pauli matrices and identity matrix respectively, it becomes

$$
\tilde{\mathcal{O}}_{z}=U^{\dagger}\left(\sigma_{z} \otimes \tau_{0}\right) U=-\sigma_{0} \otimes \tau_{y}
$$

in the original atomic orbital basis $\left(p_{x}, p_{y}, d_{x^{2}-y^{2}}, d_{x y}\right)$. Based on the pseudospin operator, we can divide the wavefunctions into two groups with positive or negative expectation values 
$\left\langle\psi_{n}(\mathbf{k})\left|\tilde{\mathcal{O}}_{z}\right| \psi_{n}(\mathbf{k})\right\rangle$ of the pseudospin. In practical calculations, we construct the pseudospin matrix at every $\mathbf{k}$ point,

$$
\begin{aligned}
O_{z}(k) & =P(\mathbf{k}) \tilde{\mathcal{O}}_{z} P(\mathbf{k}), \\
P(\mathbf{k}) & =\sum_{n=1}^{N_{\text {occ }}}\left|\psi_{n}(\mathbf{k})\right\rangle\left\langle\psi_{n}(\mathbf{k})\right|,
\end{aligned}
$$

where $\psi_{n}(\mathbf{k})$ is the $n$-th Bloch wavefunction at $\mathbf{k}$, and the summation runs over the occupied subspace. By solving the eigenvalue problem

$$
O_{z}(\mathbf{k})\left|\psi_{n}^{ \pm}(\mathbf{k})\right\rangle=s_{n}^{ \pm}\left|\psi_{n}^{ \pm}(\mathbf{k})\right\rangle
$$

one can divide the wavefunction into two groups with positive or negative pseudospin eigenvalues. For each group with $N_{\text {occ }} / 2$ bands, one then calculates the Chern number,

$$
C_{ \pm}=\frac{1}{2 \pi} \oint \operatorname{Tr}\left[\Omega_{ \pm}(\mathbf{k})\right] d k_{x} d k_{y}
$$

where $\Omega_{ \pm}(\mathbf{k})$ are the Berry curvatures for the occupied bands of the two subspaces. For the aforementioned system, the calculated pseudospin Chern number is $C_{ \pm}=\mp 1$, which is consistent with the evolution of Wannier charge center, as shown in Fig. 2(b) of the main text. This indicates the existence of nontrivial topology in the system, even though neither electron spin nor spin-orbit coupling is included.

\section{Higher-order topology from boundary perspective}

Staring from the BHZ-like Hamiltonian in Eq. (14) which supports gapless helical edge states protected by pseudo-TR, and consider pseudo-TR-breaking perturbations such as the $\Delta$ term and other terms induced at the boundary, we are now analyzing the higher-order topology of the system from the boundary perspective. To facilitate the analysis, we assume that the $C_{n}$ rotation symmetry and $n$ vertical mirror planes $\mathcal{M}_{v}$ are also preserved.

Without loss of generality, the effective Hamiltonian can now be expressed as

$$
H_{\mathrm{eff}}=\left(M-B k^{2}\right) \tau_{z}-A k_{x} \tau_{y}+A k_{y} \sigma_{z} \otimes \tau_{x}+H_{\Delta}
$$

where the first three terms represent the BHZ-like Hamiltonian. The last term $H_{\Delta}$ represents pseudo-TR-breaking perturbations. For systems with different $C_{n}$ rotation symmetries, this term has different forms. 
To study the boundary states evolution, we consider an open boundary condition at $x=0$, with the effective Hamiltonian Eq. (21) defined on the half space $x>0$ in the $x-y$ plane. For such a semi-infinite system, $k_{x}$ needs to be replaced by the operator $-i \partial_{x}$, and $k_{y}$ is still a good quantum number. As a starting point, we firstly investigate the edge states for the Hamiltonian (21) without $H_{\Delta}$ :

$$
\begin{aligned}
H_{\mathrm{eff}}\left(-i \partial_{x}, k_{y}\right)= & \left(M+B \partial_{x}^{2}\right) \tau_{z}+i A \partial_{x} \tau_{y} \\
& -B k_{y}^{2} \tau_{z}+A k_{y} \sigma_{z} \otimes \tau_{x}
\end{aligned}
$$

For $k_{y}=0$, the second line vanishes and the wave equation is given by

$$
\left[\left(M+B \partial_{x}^{2}\right) \tau_{z}+i A \partial_{x} \tau_{y}\right] \psi_{0}(x)=E \psi_{0}(x)
$$

As the wave equation (23) has particle-hole symmetry, we expect that a special edge state with $E=0$ can exist. Assuming that $\psi_{0}(x)=\phi e^{\lambda x}$, Eq. (23) can be simplified to

$$
\left[\left(M+B \lambda^{2}\right) \tau_{x} \phi=-A \lambda \phi\right.
$$

therefore the two-component wave function $\phi$ should be an eigenstate of the Pauli matrix $\tau_{x}$, i.e., $\tau_{x} \phi_{s}=s \phi_{s}(s= \pm)$. And Eq. (24) reduces to a quadratic equation for $\lambda$, which leads to $\lambda_{1,2}=\frac{A}{2 B} \pm \frac{\sqrt{A^{2}-4 M B}}{2 B}$. As we are looking for the solution with the boundary condition $\phi(x=0)=\phi(x \rightarrow+\infty)=0$, if we assume $A / B>0$, then we find only the solution with $s=+$ satisfies this boundary condition. Therefore, the general solution is given by

$$
\psi_{0}(x)=c\left(e^{-\lambda_{1} x}-e^{-\lambda_{2} x}\right) \phi_{+},
$$

where $c$ is the normalization factor. The effective edge model can be obtained by projecting the bulk Hamiltonian onto the edge states

$$
\Psi_{\uparrow}(x)=\left(\begin{array}{c}
\psi_{0} \\
\mathbf{0}
\end{array}\right), \quad \Psi_{\downarrow}(x)=\left(\begin{array}{c}
\mathbf{0} \\
\psi_{0}
\end{array}\right) .
$$

To leading order in $k_{y}$, we arrive at the 1D edge model:

$$
H_{\text {edge }}=A k_{y} \sigma_{z}
$$

indicating the existence of a pair of pseudospin polarized gapless edge states protected by the pseudo-TR symmetry. 
Now, we consider the effect of the pseudo-TR-breaking term $H_{\Delta}$ on the edge states. Using the perturbation theory, the effective edge model can be obtained by projecting $H_{\Delta}$ onto the edge states (26). The procedure leads to the $2 \times 2$ effective Hamiltonian defined by

$$
H_{\text {edge }}=A k_{y} \sigma_{z}+H_{m}
$$

where $H_{m}^{\mu \nu}=\left\langle\Psi_{\mu}\left|H_{\Delta}\right| \Psi_{\nu}\right\rangle \quad(\mu, \nu=\uparrow, \downarrow)$. As an example, we again consider the periodic approximant with $C_{4 v}$ symmetry and assume the pseudo-TR-breaking perturbation is

$$
H_{\Delta}=\sigma_{x} \otimes\left(\begin{array}{cc}
\alpha\left(\cos k_{x}-\cos k_{y}\right) & 0 \\
0 & \delta\left(\cos k_{x}+\cos k_{y}\right)
\end{array}\right) .
$$

The resulting mass term of the $1 \mathrm{D}$ edge model is given by

$$
H_{m}=m \sigma_{x}
$$

namely, the pseudo-TR-breaking term induces an effective mass term. As a result, the edge states that linearly cross at the $\Gamma$ point of the edge BZ and are captured by a $1 \mathrm{D}$ Dirac model (27), is gapped by the mass term (30). Such a gapped Dirac model admits a $Z_{2}$ topological classification by the sign of the mass term, resembling the case in the topological Su-Schrieffer-Heeger model. Therefore, a 0D bound state should appear at the corner shared by two edges with masses of opposite signs, corresponding to the Jackiw-Rebbi topological domain-wall states [15].

Next, let's consider edges connected by certain symmetries. For a conventional electronic system hosting a QSH state described by the BHZ model, the fourfold rotation operator and diagonal mirror operator are given by $\hat{C}_{4}=e^{-i \pi / 4 \sigma_{z}} \otimes \tau_{0}$ and $\hat{\mathcal{M}}_{x y}=e^{-i \pi \hat{\boldsymbol{n}} \cdot \boldsymbol{\sigma}} \otimes \tau_{0}$. However, as the pseudospin is represented by angular orbital momentum in our system, the actual symmetry is represented differently,

$$
\begin{aligned}
\hat{C}_{4}= & \left(\begin{array}{cccc}
-i & 0 & 0 & 0 \\
0 & -1 & 0 & 0 \\
0 & 0 & i & 0 \\
0 & 0 & 0 & -1
\end{array}\right), \\
\hat{\mathcal{M}}_{x y}= & \left(\begin{array}{cccc}
0 & 0 & i & 0 \\
0 & 0 & 0 & -1 \\
-i & 0 & 0 & 0 \\
0 & -1 & 0 & 0
\end{array}\right) .
\end{aligned}
$$


The full effective Hamiltonian (21) is invariant under these symmetry operators:

$$
\begin{gathered}
\hat{C}_{4} H\left(k_{x}, k_{y}\right) \hat{C}_{4}^{-1}=H\left(-k_{y}, k_{x}\right), \\
\hat{\mathcal{M}}_{x y} H\left(k_{x}, k_{y}\right) \hat{\mathcal{M}}_{x y}^{-1}=H\left(k_{y}, k_{x}\right) .
\end{gathered}
$$

As the bulk model is invariant under $\mathcal{M}_{x y}$ mirror reflection, the boundary at $y=0$ is therefore related to the aforementioned one at $x=0$ by $\mathcal{M}_{x y}$. And the effective edge model can be constructed by performing the mirror operation to the edge at $x=0$. Consequently, the edge states at the boundary $y=0$ are expressed as

$$
\begin{aligned}
& \Psi_{\downarrow}^{\prime}=\hat{\mathcal{M}}_{x y} \Psi_{\uparrow} \propto\left(\begin{array}{c}
0 \\
-\phi_{-}^{y}
\end{array}\right), \\
& \Psi_{\uparrow}^{\prime}=\hat{\mathcal{M}}_{x y} \Psi_{\downarrow} \propto\left(\begin{array}{c}
\phi_{+}^{y} \\
0
\end{array}\right),
\end{aligned}
$$

where $\phi_{ \pm}^{y}$ is the eigenstate of the Pauli matrix $\tau_{y}$, i.e., $\tau_{y} \phi_{ \pm}^{y}= \pm \phi_{ \pm}^{y}$. Based on these edge states, the effective $1 \mathrm{D}$ edge model for the boundary $y=0$ can be obtained via $H_{\text {edge }}^{\mu \nu}=\left\langle\Psi_{\mu}^{\prime}\left|H_{\text {eff }}\left(k_{x},-i \partial_{y}\right)\right| \Psi_{\nu}^{\prime}\right\rangle \quad(\mu, \nu=\uparrow, \downarrow)$ To leading order in $k_{x}$, we arrive at the effective Hamiltonian for the helical edge states:

$$
H_{\text {edge }}=-\left(A k_{x} \sigma_{z}-m \sigma_{x}\right) .
$$

Namely, these two edges have mass terms with opposite signs. Since two edges related by mirror symmetry $\mathcal{M}_{x y}$ have opposite mass terms, a corner state would generally appear at the intersection between the two edges which belong to distinct topological classes. Due to the $C_{4}$ rotation symmetry, other corners in the finite square sample would also have corner states.

For a finite sample with open boundary conditions in both $x$ and $y$ directions, $k_{x}$ and $k_{y}$ are no longer good quantum number. Instead, we introduce a real-space coordinate $l$ counterclockwise running along the boundary. Since the one-dimensional boundary is a closed loop, the definition of $l$ implies that $l+2 L_{x}+2 L_{y}=l$, where $L_{x}$ and $L_{y}$ are the size of the sample along the $x$ and $y$ directions. Using the boundary coordinates, the effective Hamiltonian on different boundaries can be written uniformly as

$$
H_{\text {edge }}=-i v(l) \partial_{l} \sigma_{z}+m(l) \sigma_{x}
$$


Here $v(l)=A$, while $m(l)$ changes sign at adjacent edges, implying the existence of domainwall states at the corners according to the Jackiw-Rebbi theory [15].

The above analysis demonstrates that the pseudo-TR-breaking perturbation with respect to $C_{4 v}$ symmetry can transform a pseudo-TR-protected QSH-like state into a higher-order topological state with topological corner modes. The discussion is quite general, not only applicable to other systems with $C_{4 v}$ symmetry but also extendable to other systems with different $C_{n v}$ symmetries including both crystalline and quasicrystalline lattices.

In general, in the symmetric finite lattice with $C_{n v}$ symmetry, the pseudo-TR-breakinginduced mass term that gaps out any boundary state should be odd under vertical mirror symmetry $\mathcal{M}_{v}$. Therefore, adjacent edges related by $\mathcal{M}_{v}$ would generally have opposite signs of mass terms, i.e., the two edges belong to different topological classes, ensuring the existence of corner states at the intersection between them. For a symmetric sample with an even number of edges, the edges are connected by the $C_{n}$ rotational symmetry, and adjacent edges are related by the $\mathcal{M}_{v}$ mirror symmetry. Therefore, the mass terms of these edges change sign alternatively across the perimeter of the sample, as shown in Fig. S10. This gives rise to topological corner states at intersections between adjacent edges. Our numerical calculations demonstrate that topological corner states indeed exist on finite symmetric samples of different lattices including rhombic, trigonal, square, honeycomb, and quasicrystalline lattices, as shown in Figs. S17-S19 and Fig. 1 and 2 of the main text.

\section{E. Mirror-graded Berry phase}

In addition to the Jackiw-Rebbi mechanism between adjacent edges, the corner states can be further understood in terms of mirror-graded Berry phases. For simplicity, we utilize the diagonal vertical mirror symmetry $\mathcal{M}_{x y}$ of the above approximants as an example. As the lattice is symmetric under $\mathcal{M}_{x y}$, wavefunctions in the mirror-invariant line $\left(k_{x}=k_{y}\right)$ can be divided into two mirror subspaces with opposite $\mathcal{M}_{x y}$ eigenvalues $( \pm)$. For each mirror subspace one can evaluate its Berry phase for all occupied states, given by

$$
\varphi_{ \pm}=i \oint_{k_{x}=k_{y}} \operatorname{Tr}\left[\mathcal{A}_{ \pm}(\mathbf{k})\right] \cdot d \mathbf{k}
$$

where $\mathcal{A}_{ \pm}(\mathbf{k})$ is the Berry connection for the occupied states in the mirror subspace \pm . Connected with the aforementioned double band inversion, the calculated $\varphi_{ \pm}=\mp \pi$ implies 

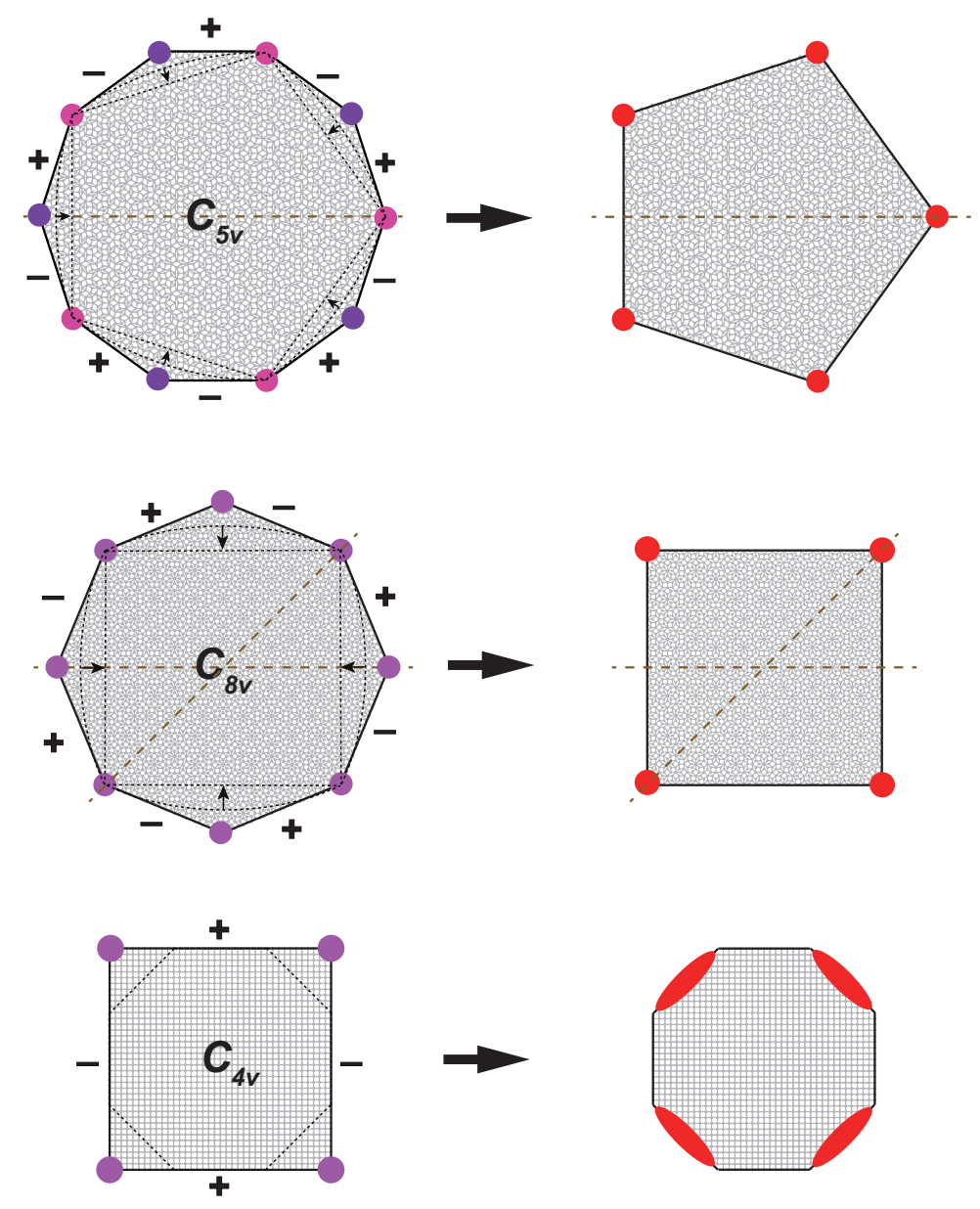

Figure S10: Schematic illustration of the evolution of topological corner states. The distribution of edge mass terms and corner states across the perimeter of (left panel) fivefold and eightfold quasicrystals, and square lattice. (right panel) By smoothly tuning the shape of the sample, the sign distribution of edge mass terms and the bounded states persist.

the existence of corner modes at intersections between edges connected by $\mathcal{M}_{x y}$. As the corner states in our proposed model depend on lattice termination (see Fig. S16 and S23), they are considered "extrinsitc", according to the topological classification of Geier et al. [16]. Because the extrinsic, termination-dependent classification of corner states in a mirrorsymmetric corner of a $2 \mathrm{D}$ lattice is the same as that of end states of a $1 \mathrm{D}$ lattice with a transverse mirror symmetry with $d_{\|}=0$ [16], the corner states in our model resemble the end modes of the topological Su-Schrieffer-Heeger model [17].

Moreover, it is found that the mirror-graded Berry phase $\varphi_{ \pm}$in our proposed models is determined by whether the crucial double band inversion occurs or not, implying the 
importance and generality of the double band-inversion mechanism for the realization of higher-order topology. As the double band inversion takes place at the center of the BZ, similar arguments are applicable to other lattices with vertical mirror symmetries, such as rhombic, trigonal, honeycomb, and quasicrystallie lattices (see Figs. S17-S19 and Figures in the main text). And the conclusion that corner states emerge at the intersections between edges connected by vertical mirror symmetries is still valid for these systems. For a symmetric sample with $C_{n v}$ symmetry, corners on $n$ vertical mirror planes are connected by the $C_{n}$ rotation symmetry. Therefore, corners are related to each other symmetrically. As a consequence, topological 0D states appear at all corners, indicating the nontrivial higher-order topology in these systems.

\section{F. Calculation of the higher-order topological invariant}

Alternatively, one can simply calculate the quadrupole moment to determine the higherorder topology of the above approximant. Taking advantage of the inversion and $C_{4}$ rotation symmetry, the bulk dipole $P_{x, y}$ and quadrupole moment $Q_{x y}$ can be obtained by checking the parity at high-symmetry points $[18,19]$,

$$
\begin{gathered}
P_{i}=\frac{1}{2}\left(\sum_{n} q_{i}^{n} \text { modulo2 }\right), \quad Q_{i j}=\sum_{n} p_{i}^{n} p_{j}^{n} \\
p_{i}^{n}=\frac{1}{2}\left(q_{i}^{n} \text { modulo2 }\right), \quad(-1)^{q_{i}^{n}}=\frac{\eta^{n}\left(X_{i}\right)}{\eta^{n}(\Gamma)}
\end{gathered}
$$

where $\eta^{n}\left(X_{i}\right)$ is the parity of $n$-th occupied band at the $X_{i}$ point, $i$ stands for $x$ or $y$, and $q_{i}^{n}$ equals either 0 or 1 . The calculated bulk dipole and quadrupole moment are $P_{x}=P_{y}=0$ and $Q_{x y}=1 / 2$, confirming the nontrivial higher-order topology in the system. The same quadrupole moment is also obtained for other periodic approximants with increasing lattice size when $p$-d band inversions occur, indicating the existence of higher-order topological states in all these systems.

\section{G. Discussion of fivefold quasicrystals}

The above analysis demonstrates that topological corner states exist in symmetric samples with an even number of edges and corners as the argument relies on an alternating sign of the mass term at the boundary. Therefore, it is not applicable to the symmetric 


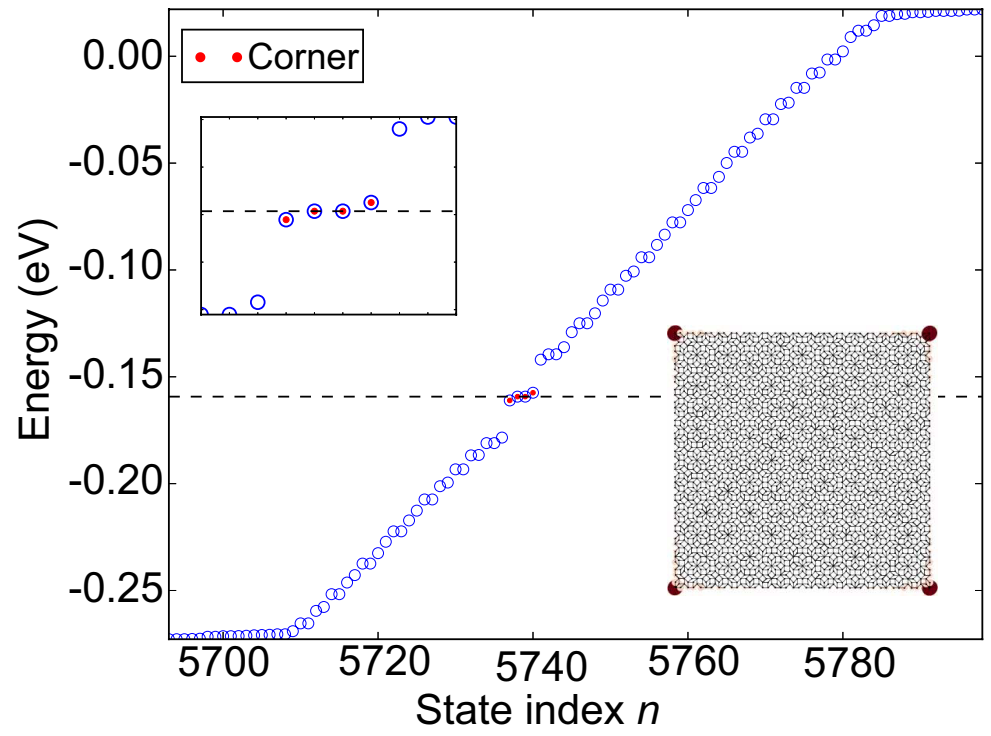

Figure S11: Corner states of a square-shaped sample of the Ammann-Beenker-type quasicrystal. The inset shows the spatial intensity distribution of in-gap corner states.

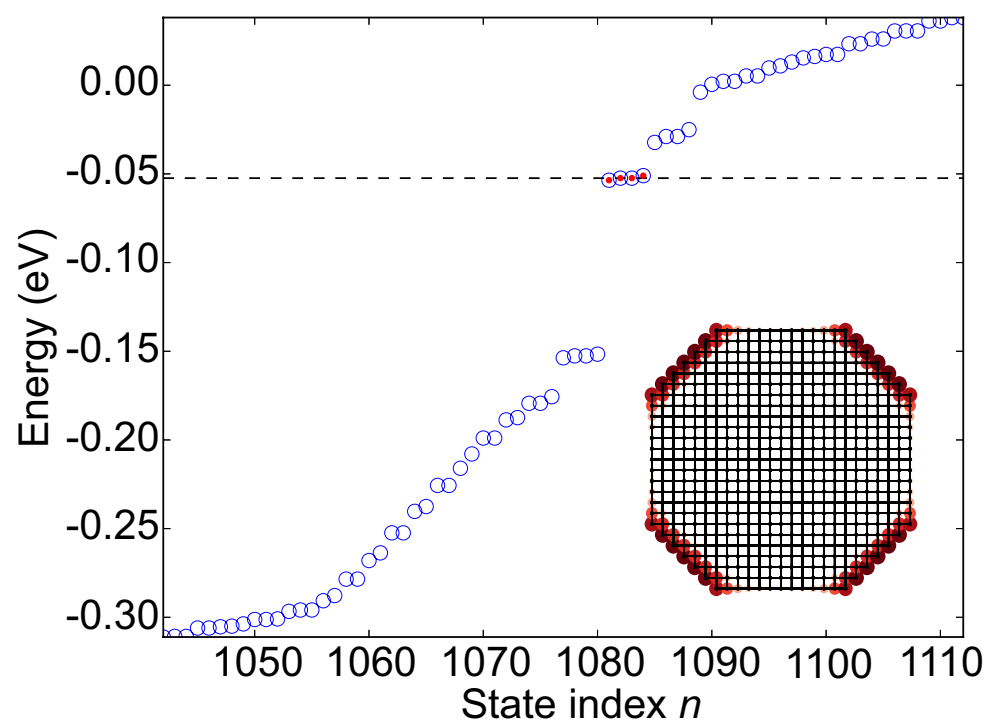

Figure S12: Extended corner states (marked in red) of a truncated-square sample of the square lattice. The inset shows the spatial intensity distribution of extended corner states.

pentagonal sample of fivefold quasicrystals. However, based on a transformation process of the boundary, one can validate the existence of topological states at ORS corners.

Hypothetically, let's start with a decagonal sample of the fivefold quasicrystal, as shown in Fig. S10. Following the general argument, the pseudo-TR-breaking-induced mass term which gap out boundary states is odd under vertical mirror symmetry. As there are five vertical 
mirror planes, mass terms of the ten edges change sign alternatively across the perimeter of the sample. Because the sample only possesses the $C_{5}$ symmetry (not $C_{10}$ ), corners at opposite ends are not centrosymmetric, which leads to different energies of corresponding corner states as denoted in different colors in Fig. S10. Now, let's continually move the boundary to form a pentagonal sample, i.e., smoothly moving alternative five of the ten corners towards the center of the sample. As the edges of the pentagonal sample become mirror symmetric, the domain-wall argument fails to apply as mass terms are not necessarily generated in the two sides of the mirror-symmetric edge [16, 20-22]. Therefore, the domainwall state at the middle of the edge disappear, leaving only five corner states still persist at the Fermi level. Since the HOTQI is attributed to the double band inversion mechanism, the mirror symmetries that facilitate the analysis of corner state are not essential for their existence. The remaining five corner states thus are still compatible with the quasicrystalline rotational symmetry of the fivefold quasicrystal. Despite different number of corner states in different $C_{5 v}$-symmetric samples, the corner-induced filling anomaly [10-12, 23] is still valid in all of them, which guarantees the existence of at least one set of fivefold degenerate states localized at corners and the realization of topological corner charge in $C_{5 v}$-symmetric quasicrystals.

Similar transformation processes are also applicable to eightfold quasicrystals to analyze the evolution of corner states in square samples of the Ammann-Beenker-type quasicrystal (see Fig. S10). As shown in Fig. S11, for the square-shaped sample of the Ammann-Beenkertype eightfold quasicrystal, only four corner states appear around the Fermi level in the energy spectrum. On the contrary, if one cut four corners of a square sample of the square lattice to realize an octagonal-shape sample with eight corners, instead of the emergence of eight corner states, four original corner states would spread among the truncated corners, as illustrated in Fig. S10. In the truncated-square sample of the square lattice, adjacent edges are not symmetric under any vertical mirror symmetries. Instead, interval edges are connected by a diagonal vertical mirror symmetry and have opposite signs of mass terms. Therefore, four extended corner states persist in the new truncation-induced edges, as shown in Fig. S12. 

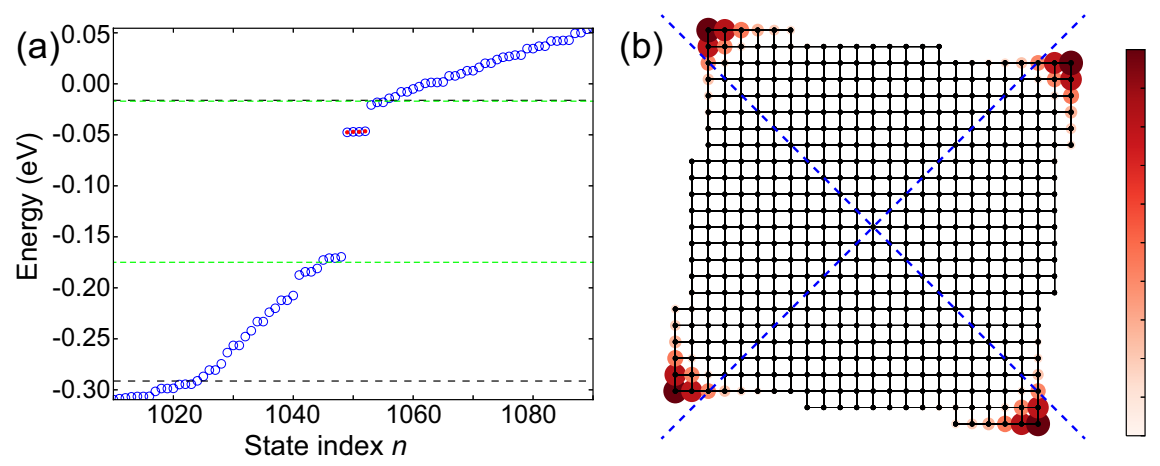

Figure S13: Topological corner states in an asymmetric sample of the square lattice. The localized corner states persist at the intersection between two edges that cannot be related by any vertical mirror symmetry. The reflection line is shown dashed.

(a)
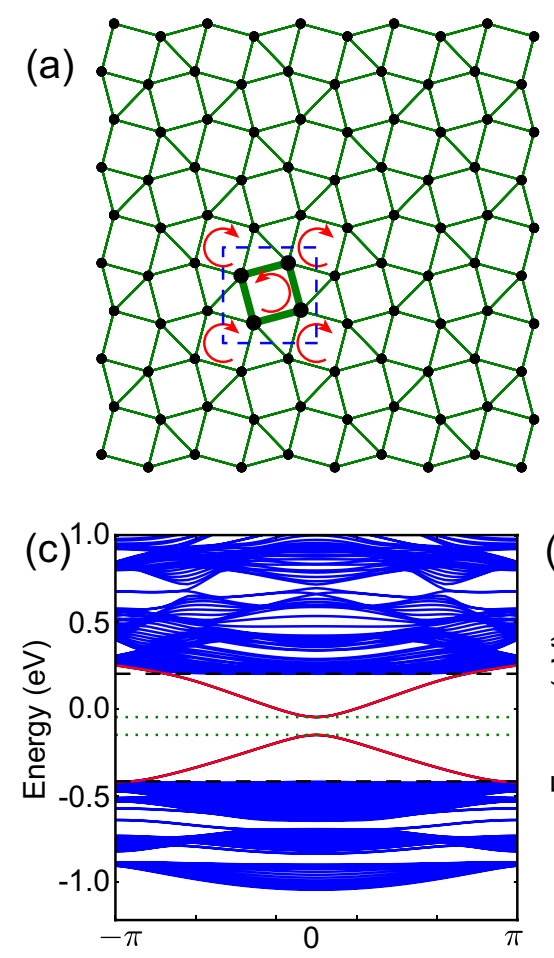
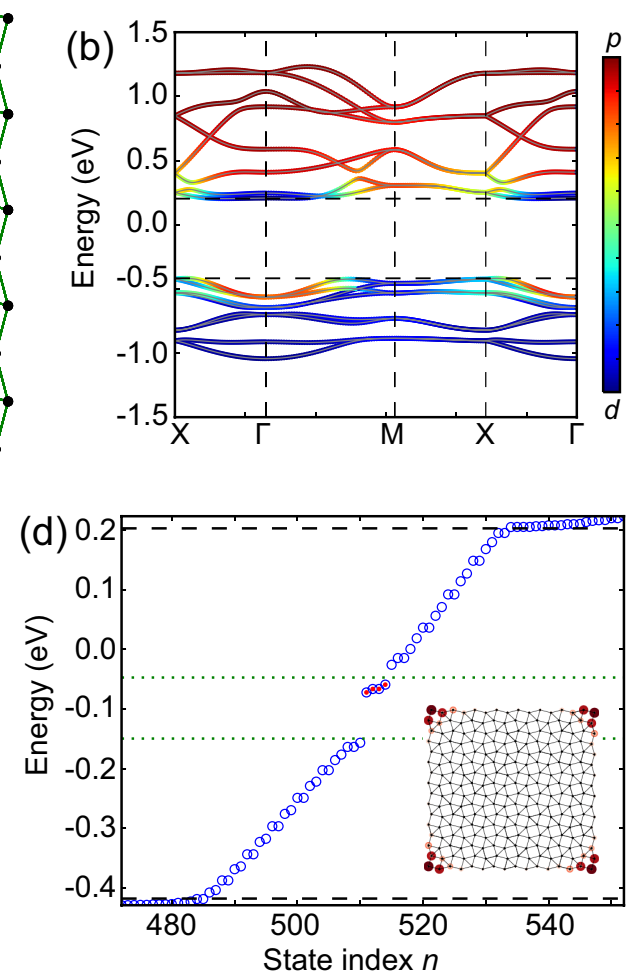

Figure S14: Higher-order topological state in a snub-square lattice without any vertical mirror symmetry. (a) The lattice structure of the snub-square lattice. The rotational angle is $15^{\circ}$. (b) Orbital-resolved bulk band structure where a $p$ - $d$ band inversion occurs around $\Gamma$. (b) Band structure of a nanoribbon where the topological edge states (the red lines) are gapped. (d) Energy spectrum of a square nanodisk of the snub-square lattice. The inset shows the spatial intensity distribution of in-gap corner states. 


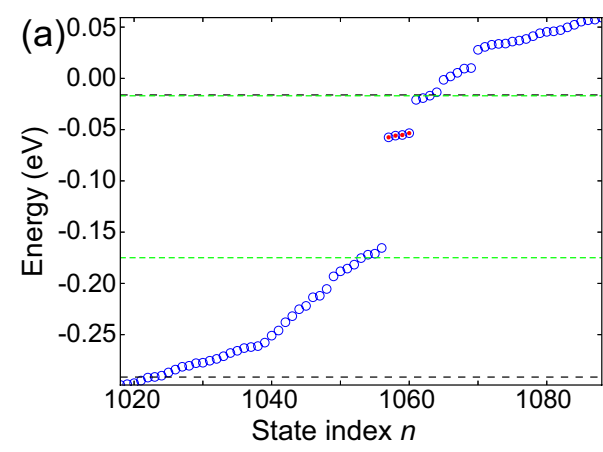

(b)

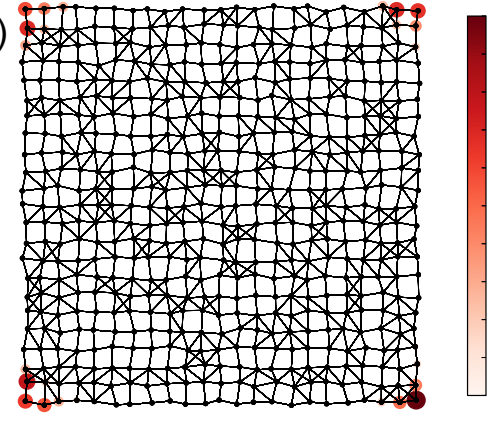

Figure S15: Robustness of topological corner states against random displacements in a finite sample of the square lattice. The localized corner states persist at four corners of the random lattice without $C_{4}$ rotation or vertical mirror symmetry.

\section{H. Robustness of topological corner states}

The HOTQI is attributed to the double band inversion mechanism. Based on the generic argument from the symmetry analysis and the boundary perspective, which clarified the topological origin of the corner states, we conclude that higher-order topology would occur in different systems. Although we have assumed the vertical mirror symmetry above to facilitate the analysis, in fact, these symmetries are not strictly required for the existence of the topological 0D states [24]. Because the topological classification for each edge is not expected to be changed by any perturbations that weakly break the symmetry. Therefore, it is important to show that the resulting corner states remain robust in the presence of weak symmetry-breaking perturbations, as long as bulk and edge gaps remain open and the original inverted band ordering doesn't change. Below, we will explicitly demonstrate this point in the examples.

Although topological corner states are localized at corners where the mirror-related edges meet for symmetric samples with $C_{n v}$ symmetry, (see, for example, Fig. S9(f) and the inset of Fig. 2(d) in the main text), the symmetry itself is not essential for the existence of the corner state. As shown in Fig. S13, the corner states persist if the finite sample is cut asymmetrically, such that there are no longer any mirror-related edges.

Next, we consider a periodic snub-square lattice (the snub-quadrille structure) with only $C_{4}$ rotation symmetry. Such a snub-square lattice can be constructed by applying a snub operation to a corner-shared square tiling with certain rotational angles. As shown in 

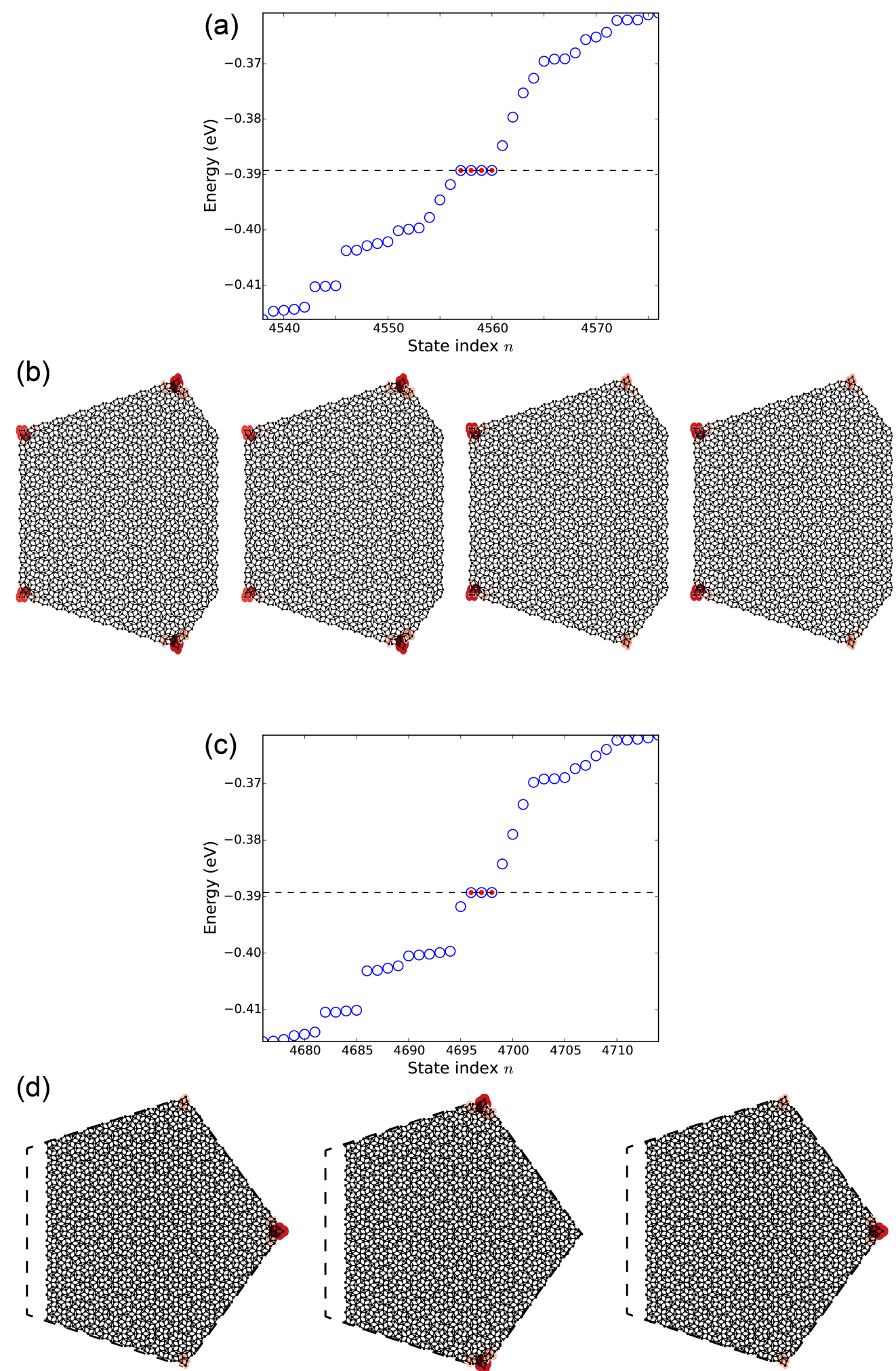

Figure S16: Energy spectrum and corner states of asymmetric samples of the fivefold quasicrystal. $(\mathrm{a}, \mathrm{b})$ The sample is constructed by removing the right corner of a pentagonal sample. (c,d) The sample is constructed by removing some atoms in the left edge of a pentagonal sample. 
Fig. S14, the localized corner modes remain robust even if the crystal is rotated locally to form a snub-square lattice where all vertical mirror symmetries are broken. This indicates that the corner states are robust against weak mirror-symmetry-breaking perturbations, namely, $\mathcal{M}_{v}$ is actually not strictly required for the existence of the topological corner states.

Lastly, we adopted a randomly distorted square lattice to investigate the robustness of the topological corner states. The distorted square lattice is generated by randomly displacing atoms away from their equilibrium positions within a radius of $r=0.3$ (assuming the original lattice constant is 1). As shown in Fig. S15, topologically protected states at corners continue to exist in the distorted square lattice where almost all crystalline symmetries are broken.

\section{HOTIs induced by $p$ - $d$ band inversion in different lattices}

The $p$ - $d$ band inversion can induce higher-order topology in various lattices with $C_{n}$ rotation and vertical mirror symmetries $\mathcal{M}_{v}$. Figure S17-S19 show HOTI states in rhombic,
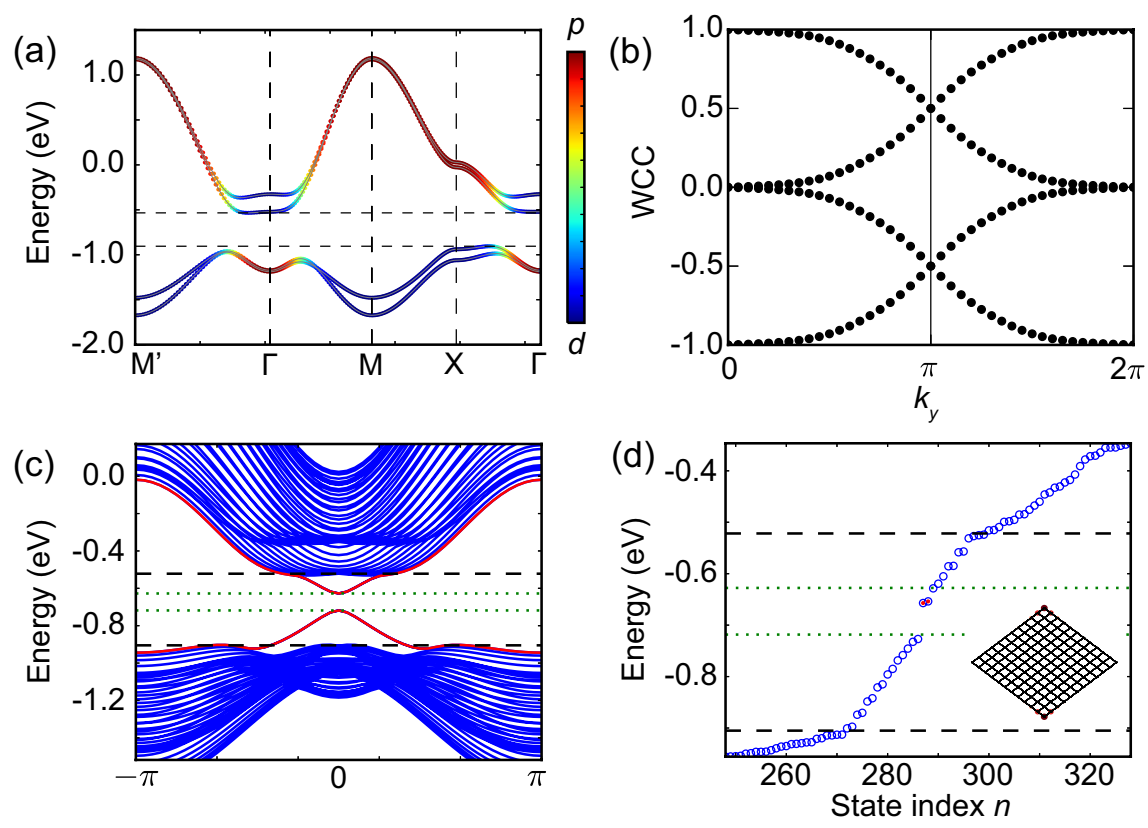

Figure S17: Higher-order topological state in a rhombic lattice. (a) The lattice structure. (b) Orbital-resolved bulk band structure where a $p$ - $d$ band inversion occurs around $\Gamma$. (c) The evolution of Wannier charge center $\langle x\rangle$ as a function of $k_{y}$. (d) Band structure of nanoribbons where the topological edge states (the red lines) are gapped. (e) Energy spectrum of a nanodisk of the rhombic lattice. The inset shows the spatial intensity distribution of in-gap corner states. 

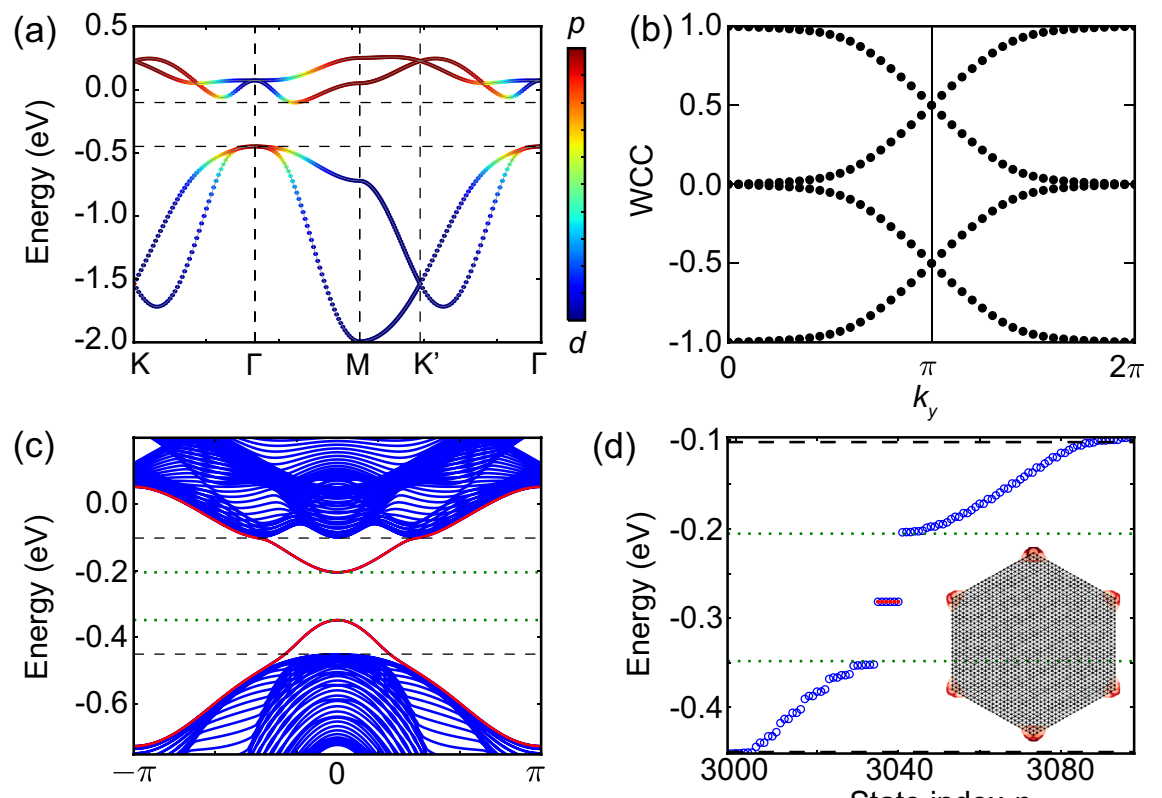

(d)

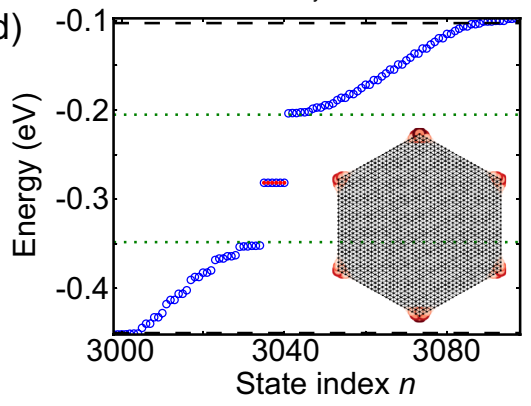

Figure S18: Higher-order topological state in a trigonal lattice. (a) The lattice structure. (b) Orbital-resolved bulk band structure where a $p$ - $d$ band inversion occurs around $\Gamma$. (c) The evolution of Wannier charge center $\langle x\rangle$ as a function of $k_{y}$. (d) Band structure of nanoribbons where the topological edge states (the red lines) are gapped. (e) Energy spectrum of a hexagonal nanodisk of the trigonal lattice. The inset shows the spatial intensity distribution of in-gap corner states.

trigonal, and honeycomb lattices, respectively.

\section{SECTION S3: HIGHER-ORDER TOPOLOGY INDUCED BY OTHER TYPE- S OF BAND INVERSION}

In addition to the double band inversion between $p$ and $d$ orbitals, we find that other types of double band inversion mechanisms, such as $d-d, d-f$, and $f-g$ band inversion [see Fig. S20, Table S1 and Table S2], are also feasible to achieve HOTQI states in quasicrystals as long as its bulk and edge gaps remain open. As the pseudospin is represented by positive and negative angular momentum, different pairs of orbitals with opposite magnetic quantum numbers can be adopted to construct HOTIs. 

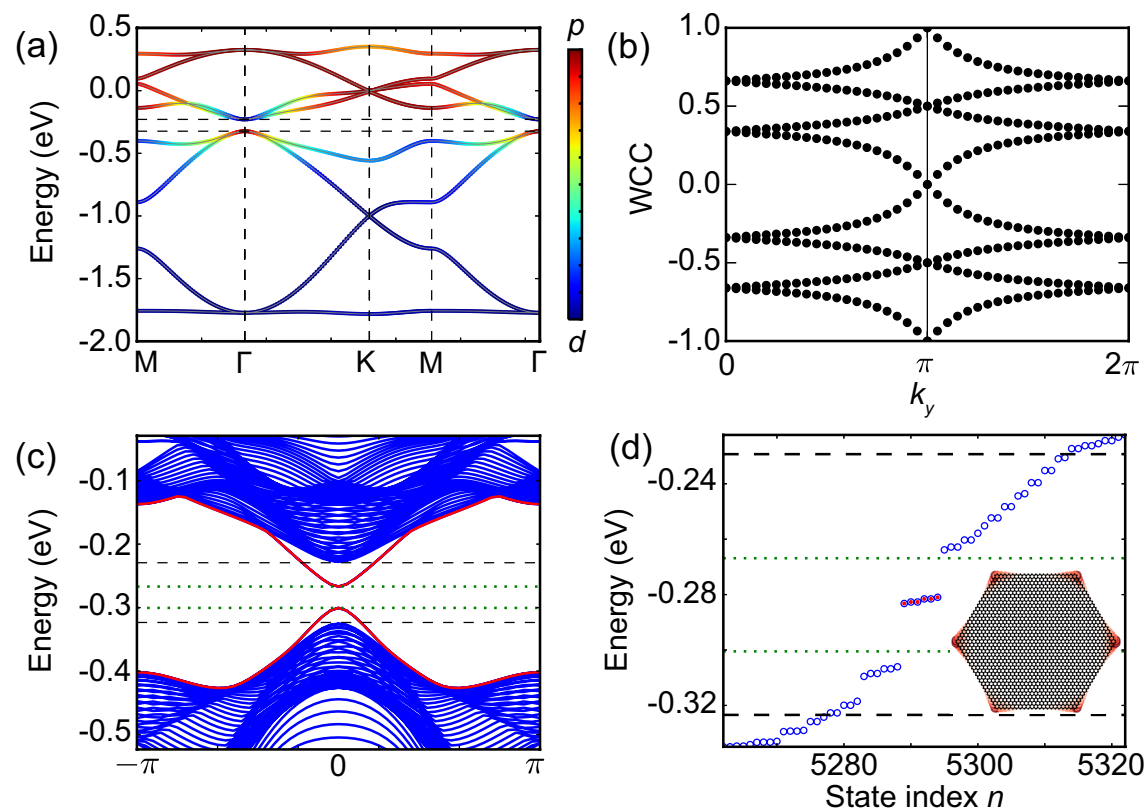

Figure S19: Higher-order topological state in a honeycomb lattice. (a) The lattice structure. (b) Orbital-resolved bulk band structure where a $p$ - $d$ band inversion occurs around $\Gamma$. (c) The evolution of Wannier charge center $\langle x\rangle$ as a function of $k_{y}$. (d) Band structure of nanoribbons where the topological edge states (the red lines) are gapped. (e) Energy spectrum of a hexagonal nanodisk of the honeycomb lattice. The inset shows the spatial intensity distribution of in-gap corner states.

\section{A. Band inversion between $d_{ \pm 1}$ and $f_{ \pm 2}$ orbitals}

As an example, we consider the double band inversion between $d_{x z} \pm i d_{y z}$ and $f_{z\left(x^{2}-y^{2}\right)} \pm$ $i f_{x y z}$ orbitals [25]. As shown in Figs. S21 and S22, the $d-f$ band inversion induces a higherorder topological insulator in the square lattice and the Ammann-Beenker-type eightfold quasicrystal as expected.

\section{B. Band inversion between $d_{ \pm 1}$ and $d_{ \pm 2}$ orbitals}

Next, we study the double band inversion between $d_{x z} \pm i d_{y z}$ and $d_{x^{2}-y^{2}} \pm i d_{x y}$ orbitals. Because $d_{x z} \pm i d_{y z}\left(d_{x^{2}-y^{2}} \pm i d_{x y}\right)$ orbitals are odd (even) under the in-plane mirror reflection, direct hoppings between these two groups are forbidden. Consequently, the bulk band cannot be gapped when the double band inversion occurs. We, therefore, introduce structural 


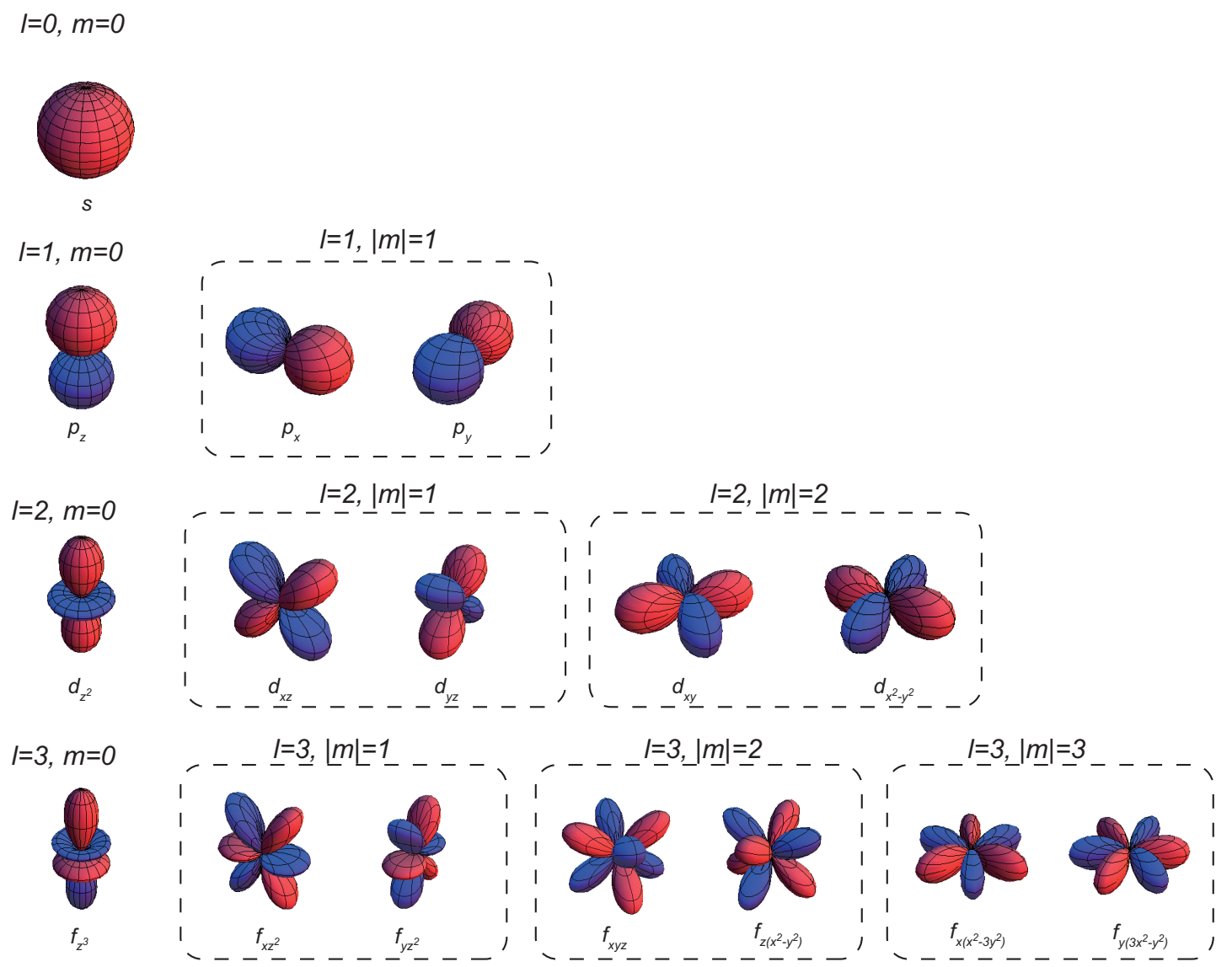

Figure S20: Atomic orbitals that can be used to construct HOTIs.

buckling to break the in-plane mirror symmetry, which then opens a gap in the bulk band. The gapped system in the buckled lattice becomes a HOTI, as shown in Fig. S25.

[1] H. Tsunetsugu, T. Fujiwara, K. Ueda, and T. Tokihiro, Phys. Rev. B 43, 8879 (1991), URL https://link.aps.org/doi/10.1103/PhysRevB.43.8879.

[2] O. Entin-Wohlman, M. Kléman, and A. Pavlovitch, Journal de Physique 49, 587 (1988).

[3] H. Tsunetsugu, T. Fujiwara, K. Ueda, and T. Tokihiro, J. Phys. Soc. Jpn. 55, 1420 (1986).

[4] M. Senechal, Quasicrystals and geometry (Cambridge University Press, 1996).

[5] N. G. De Bruijn, Kon. Nederl. Akad. Wetensch. Proc. Ser. A 43, 1 (1981).

[6] M. Duneau, R. Mosseri, and O. Christophe, J. Phys. A: Math. Gen. 22, 4549 (1989).

[7] B. Grünbaum and G. C. Shephard, Tilings and patterns (Freeman, New York, 1987).

[8] F. P. M. Beenker, Algebraic theory of non-periodic tilings of the plane by two simple build- 

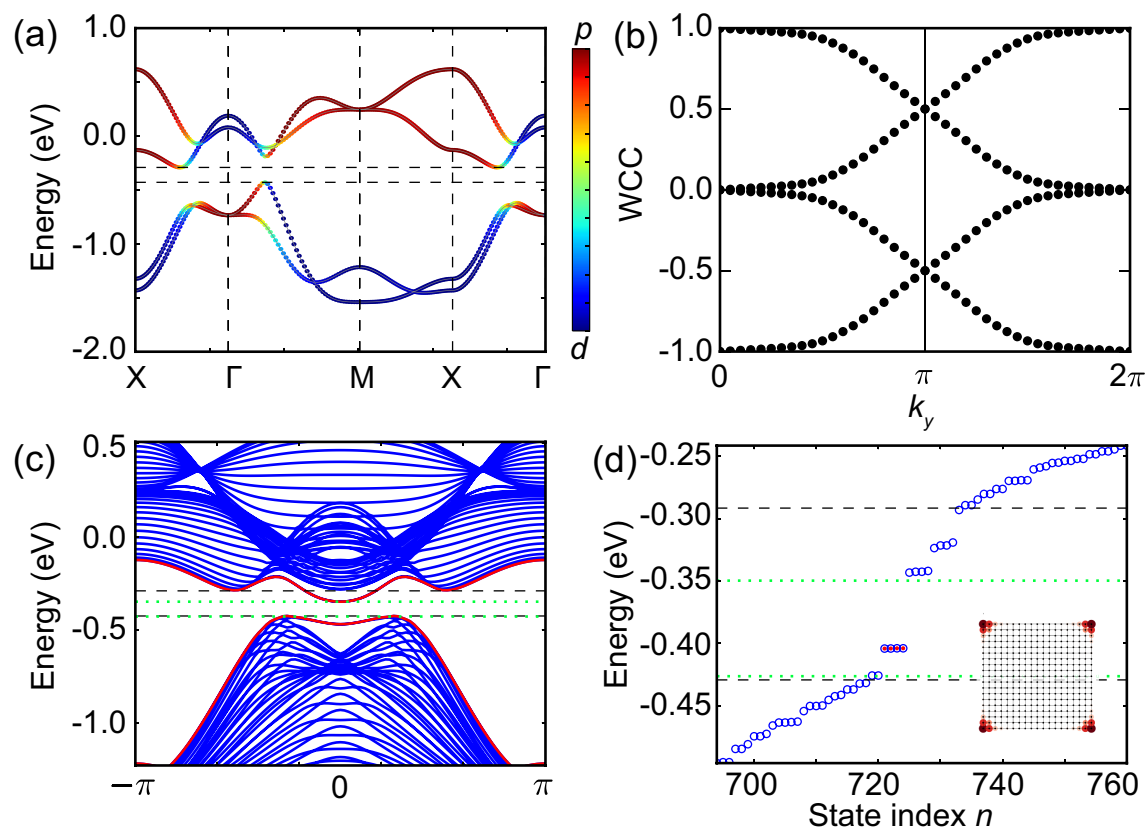

Figure S21: A $d-f$ band inversion induced higher-order topological state in the square lattice. The parameters used here are $\epsilon_{d}=0.0, \epsilon_{f}=-1.0, V_{d d \sigma}=-0.576, V_{d d \pi}=-0.0288, V_{d d \delta}=$ $-0.216, V_{f f \sigma}=0.576, V_{f f \pi}=0.173, V_{f f \delta}=0.216, V_{f f \phi}=0.144, V_{d f \sigma}=0.288, V_{d f \pi}=0.0288, V_{d f \delta}=$ $0.173 \mathrm{eV}$. (a) The band inversion between conduction and valence bands dominated by $\left(d_{x z}, d_{y z}\right)$ and $\left(f_{z\left(x^{2}-y^{2}\right)}, f_{x y z}\right)$ occurs around $\Gamma$. (b) Orbital-resolved bulk band structure where a $d$ - $f$ band inversion occurs around $\Gamma$. (c) The evolution of Wannier charge center $\langle x\rangle$ as a function of $k_{y}$. (d) Band structure of nanoribbons where the topological edge states (the red lines) are gapped. (e) Energy spectrum of a square nanodisk of the square lattice. The inset shows the spatial intensity distribution of in-gap corner states.

ing blocks: a square and a rhombus (Eindhoven University of Technology Eindhoven, The Netherlands, 1982).

[9] M. Baake and U. Grimm, Aperiodic Order, vol. 1 (Cambridge University Press, 2013).

[10] W. A. Benalcazar, T. Li, and T. L. Hughes, Phys. Rev. B 99, 245151 (2019), URL https: //link.aps.org/doi/10.1103/PhysRevB.99.245151.

[11] H. Watanabe and S. Ono, Phys. Rev. B 102, 165120 (2020), URL https://link.aps.org/ doi/10.1103/PhysRevB.102.165120.

[12] R. Takahashi, T. Zhang, and S. Murakami, Phys. Rev. B 103, 205123 (2021), URL https: //link.aps.org/doi/10.1103/PhysRevB.103. 205123. 


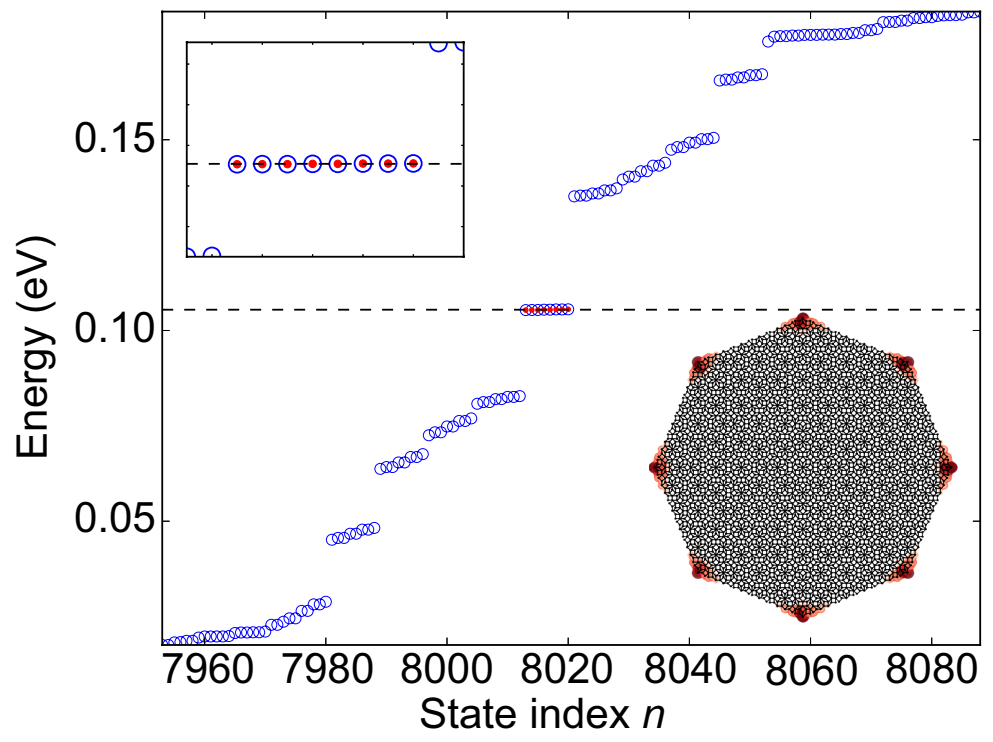

Figure S22: A $d-f$ band inversion induced higher-order topological state in the Ammann-Beenkertype eightfold quasicrystal. The parameters used here are the same as those in Fig. S21. The left inset shows the zoomed-in plot of the spectrum around the Fermi level where corner states are marked in red. The right inset shows the spatial charge distribution of corner states.

[13] B. A. Bernevig, T. L. Hughes, and S.-C. Zhang, Science 314, 1757 (2006).

[14] L.-H. Wu and X. Hu, Phys. Rev. Lett. 114, 223901 (2015), URL https://link.aps.org/ doi/10.1103/PhysRevLett.114.223901.

[15] R. Jackiw and C. Rebbi, Physical Review D 13, 3398 (1976).

[16] M. Geier, L. Trifunovic, M. Hoskam, and P. W. Brouwer, Phys. Rev. B 97, 205135 (2018), URL https://link.aps.org/doi/10.1103/PhysRevB.97.205135.

[17] W. P. Su, J. R. Schrieffer, and A. J. Heeger, Phys. Rev. Lett. 42, 1698 (1979), URL https: //link.aps.org/doi/10.1103/PhysRevLett.42.1698.

[18] F. Liu and K. Wakabayashi, Phys. Rev. Lett. 118, 076803 (2017), URL https://link.aps. org/doi/10.1103/PhysRevLett.118.076803.

[19] B. Liu, G. Zhao, Z. Liu, and Z. Wang, Nano Lett. 19, 6492 (2019).

[20] L. Trifunovic and P. W. Brouwer, Phys. Rev. X 9, 011012 (2019), URL https://link.aps. org/doi/10.1103/PhysRevX.9.011012.

[21] L. Trifunovic and P. W. Brouwer, Phys. Status Solidi B p. 2000090 (2020).

[22] L. Trifunovic, Phys. Rev. Research 2, 043012 (2020), URL https://link.aps.org/doi/10. 

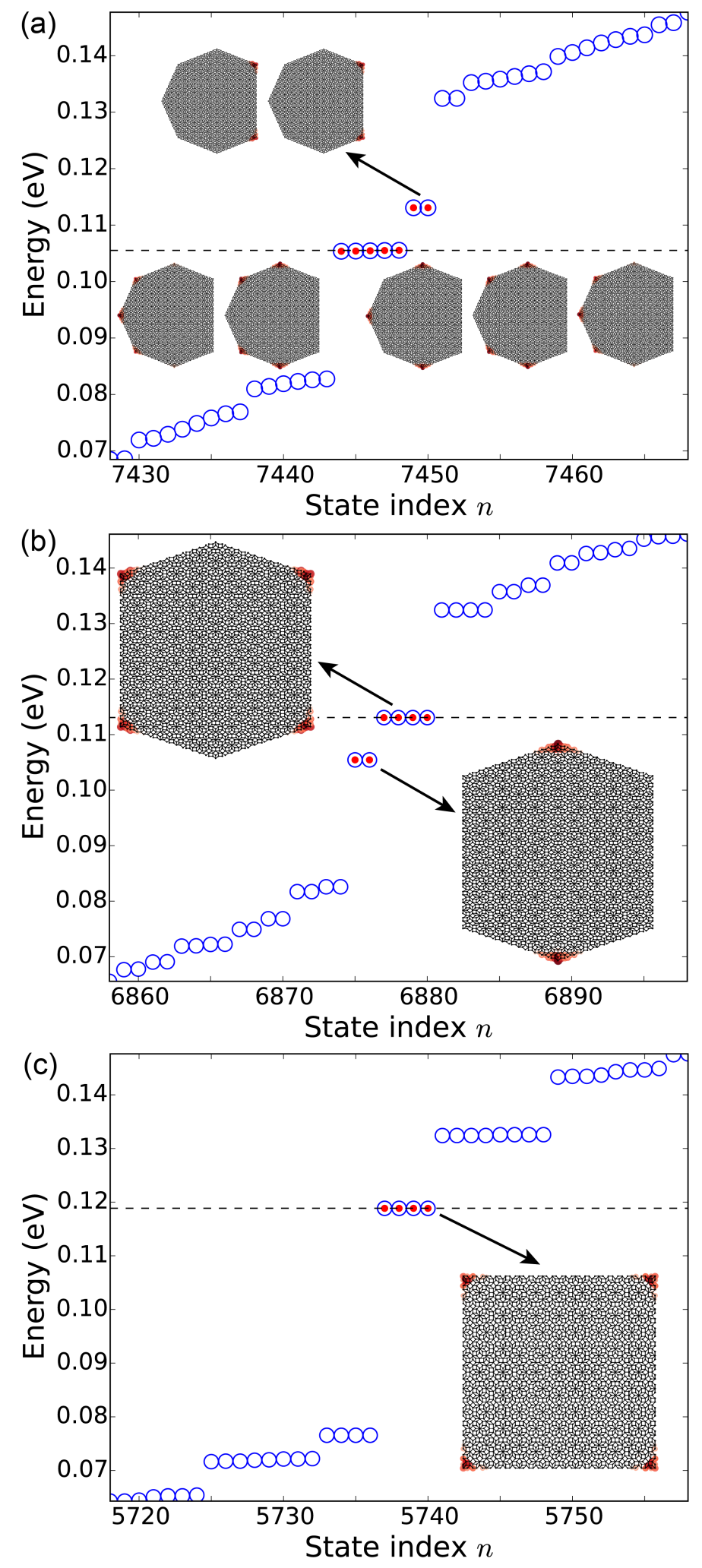

Figure S23: Corner states of asymmetric samples of the eightfold quasicrystal. The samples are constructed (a) by removing the right corner, (b) by removing the left and right corners, (c) by remove the left, right top, and bottom corners of an octagonal sample. The insets show the spatial intensity distribution of states near the Fermi level. 


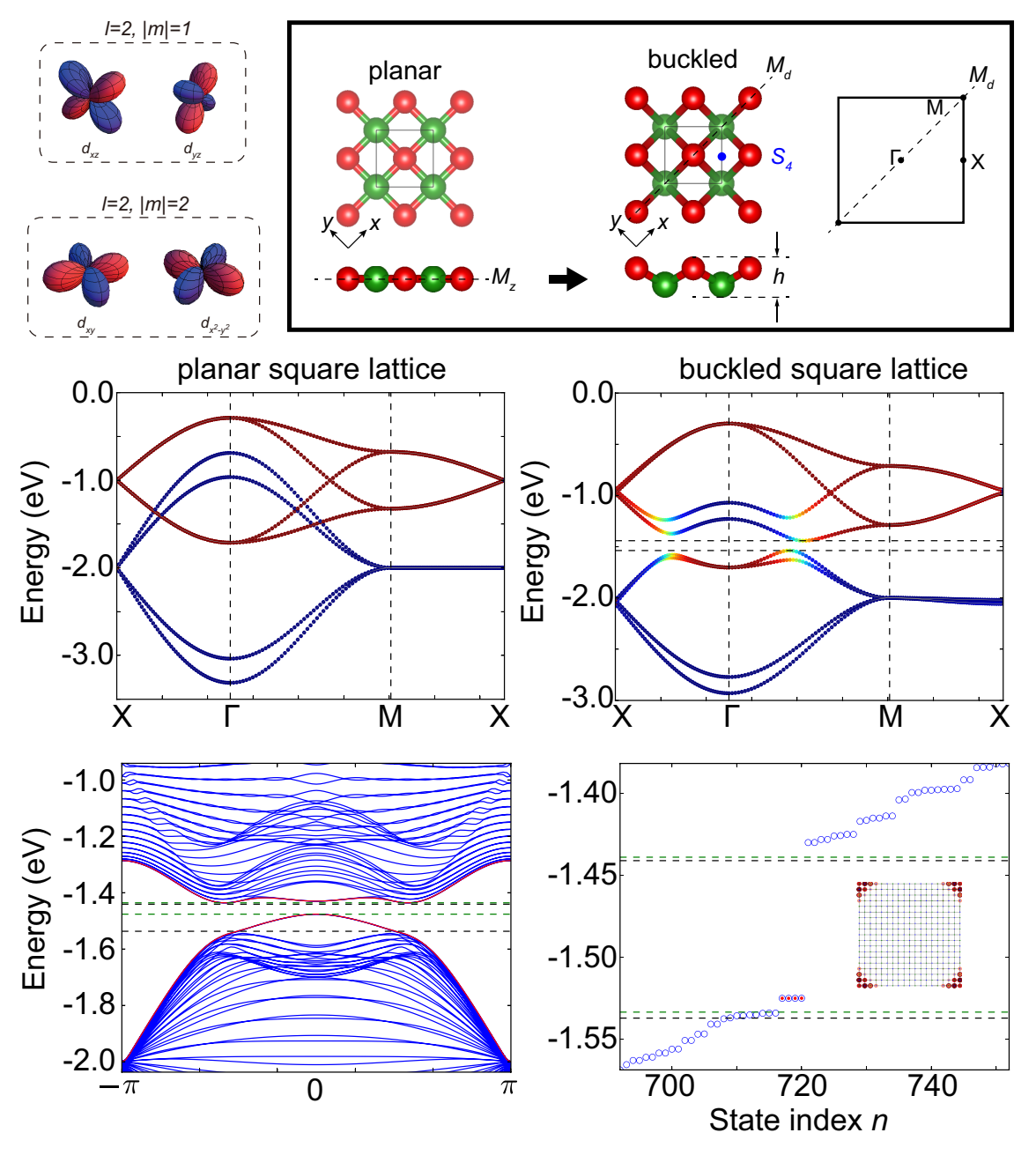

Figure S24: A $d$-d band inversion induced higher-order topological state in buckled square lattices. The parameters for the square lattice are $\epsilon_{x z, y z}=-1.0, \epsilon_{x^{2}-y^{2}, x y}=-2.0, V_{d d \sigma}=$ $-0.405, V_{d d \pi}=-0.259, V_{d d \delta}=-0.097 \mathrm{eV}$. Band inversion is between states dominated by $\left(d_{x z}, d_{y z}\right)$ and $\left(d_{x^{2}-y^{2}}, d_{x y}\right)$ orbitals. An energy gap is induced by structural buckling.

1103/PhysRevResearch.2.043012.

[23] P. M. Lenggenhager, X. Liu, T. Neupert, and T. Bzdušek, arXiv preprint arXiv:2104.11254 (2021).

[24] J. Langbehn, Y. Peng, L. Trifunovic, F. von Oppen, and P. W. Brouwer, Phys. Rev. Lett. 119, 246401 (2017), URL https://link.aps.org/doi/10.1103/PhysRevLett.119.246401.

[25] K. Takegahara, Y. Aoki, and A. Yanase, J. Phys. C: Solid State Physics 13, 583 (1980). 

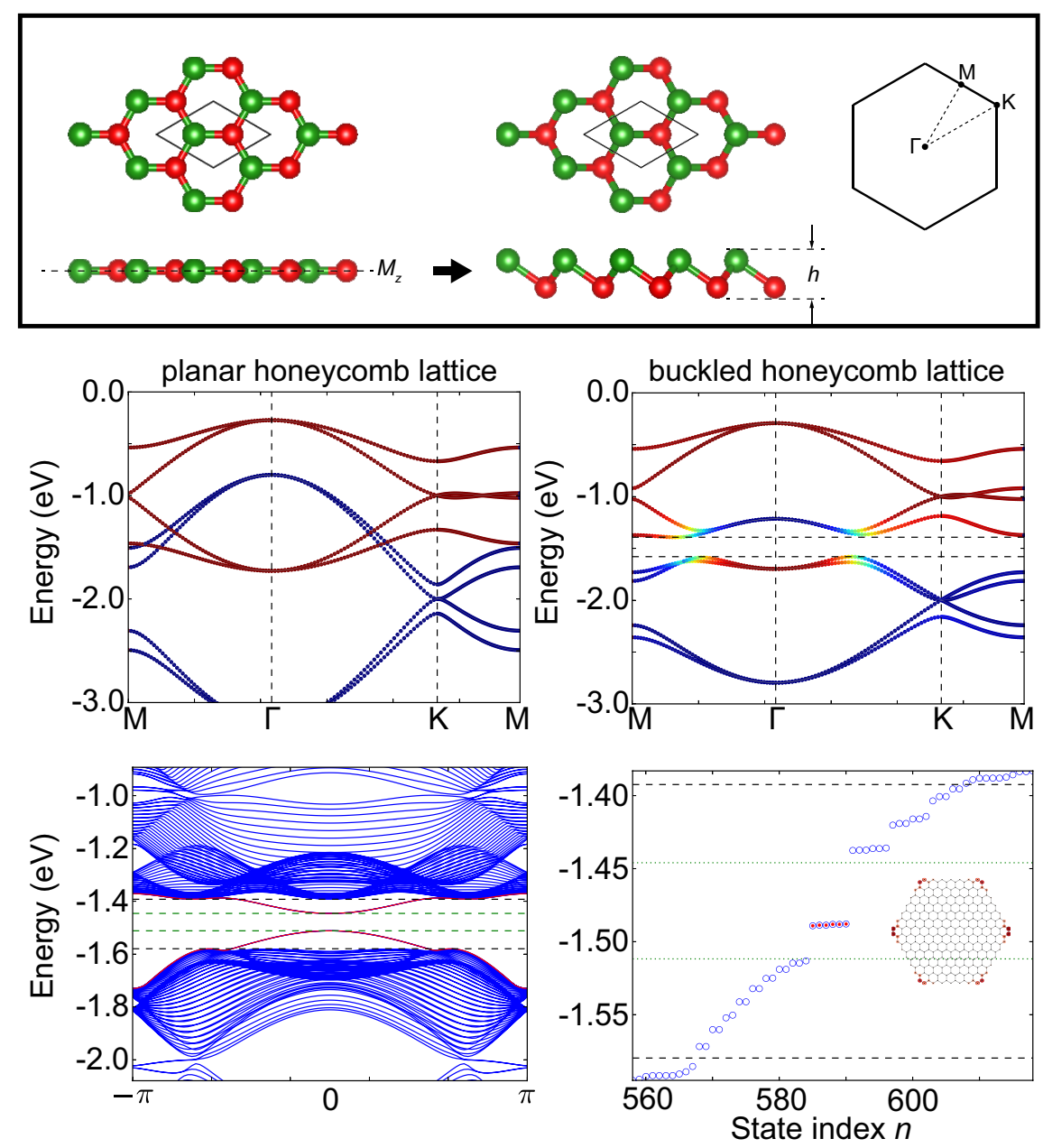

Figure S25: A $d$-d band inversion induced higher-order topological state in buckled honeycomb lattices. The parameters for the square (honeycomb) lattice are $\epsilon_{x z, y z}=-1.0, \epsilon_{x^{2}-y^{2}, x y}=$ $-2.0, V_{d d \sigma}=-0.551, V_{d d \pi}=-0.353, V_{d d \delta}=-0.132 \mathrm{eV}$. Band inversion is between states dominated by $\left(d_{x z}, d_{y z}\right)$ and $\left(d_{x^{2}-y^{2}}, d_{x y}\right)$ orbitals. An energy gap is induced by structural buckling. 